\title{
Crime and Justice
}

There are differences in the legal and judicial systems of England and Wales, Scotland and Northern Ireland which make it impossible to provide tables covering the UK as a whole in this section. These differences concern the classification of offences, the meaning of certain terms used in the statistics, the effects of the several Criminal Justice Acts and recording practices.

\section{Recorded crime statistics}

\section{(Table 11.3)}

Crimes recorded by the police provide a measure of the amount of crime committed. The statistics are based on counting rules, revised with effect from 1 April 1998, which are standard for all the police forces in England, Wales and Northern Ireland. They now include all indictable and triableeither-way offences together with a few summary offences which are closely linked to these offences. The new rules have changed the emphasis of measurement more towards one crime per victim and have also increased the coverage of offences. These changes have particularly impacted on the offences of violence against the person, fraud and forgery, drugs offences and other offences.

For a variety of reasons many offences are either not reported to the police or not recorded by them. The changes in the number of offences recorded do not necessarily accurately reflect the changes in the amount of crime committed.

In order to further improve the consistency of recorded crime statistics and to take a more victim oriented approach to crime recording, the National Crime Recording Standard (NCRS) was introduced across all forces in England, Wales and Northern Ireland with effect from 1 April 2002. Some police forces implemented the principles of NCRS in advance of its introduction across all forces. The NCRS had the effect of increasing the number of offences recorded by the police.

Similarly, the Scottish Crime Recording Standard (SCRS) was introduced by the eight Scottish police forces with effect from 1 April 2004. This means that no corroborative evidence is required initially to record a crime-related incident as a crime if so perceived by the victim. Again, the introduction of this new recording standard was expected to increase the numbers of minor crimes recorded by the police, such as minor crimes of vandalism, minor thefts, offences of petty assault and breach of the peace. However, it was expected that the SCRS would not have much impact on the figures for the more

serious crimes of serious assault, sexual assault, robbery or housebreaking.

The Sexual Offences Act 2003, introduced in May 2004, altered the definition and coverage of sexual offences. In particular, it redefined indecent exposure as a sexual offence, which is likely to account for much of the increase in sexual offences.

Further information is available from Crime in England and Wales 2007/2008 (Home Office, Sian Nicholas, Chris Kershaw and Alison Walker, editors).

\section{Court proceedings and police cautions}

\section{(Tables 11.4-11.8, 11.13-11.17, 11.20-11.22)}

The statistical basis of the tables of court proceedings is broadly similar in England and Wales, Scotland and Northern Ireland. The tables show the number of persons found guilty, recording a person under the heading of the principal offence of which he/she was found guilty, but excluding additional findings of guilt at the same proceedings. A person found guilty at a number of separate court proceedings is included more than once.

The statistics on offenders cautioned in England and Wales cover only those who, on admission of guilt, were given a formal caution by, or on the instructions of, a senior police officer as an alternative to prosecution. Written warnings given by the police for motor offences and persons paying fixed penalties for certain motoring offences are excluded. Formal cautions are not issued in Scotland. There are no statistics on cautioning available for Northern Ireland.

The Crime and Disorder Act 1998 provides for reprimands and final warnings, which are new offences and orders implemented nationally from 1 June 2000 . They replace the system of cautioning for offenders under the age of 18 . Reprimands can be given to first-time offenders for minor offences. Any further offending results in either a final warning or a charge.

For persons proceeded against in Scotland, the statistics relate to the High Court of Justiciary, the sheriff courts and the district courts. The High Court deals with serious solemn offences (requiring trial by jury) and has unlimited sentencing power. Sheriff courts deal with solemn offences where imprisonment is limited to 3 years or summary offences (not requiring a jury) where imprisonment is limited to 3 months ( 6 months when specified in legislation for second or subsequent offences and 12 months for certain statutory offences). District courts deal only with summary cases and are limited to 60 days imprisonment and level 4 fines. Stipendiary magistrates 
sit in Glasgow District Court and have the summary sentencing powers of a sheriff.

In England and Wales, indictable offences are offences which are:

- triable only on indictment. that is by the Crown Court. ('indictable-only' offences include murder, manslaughter, rape and robbery)

- triable either way. that is by the Crown Court or a magistrates' court

The Criminal Justice Act 1991 resulted in the following changes being made to the sentencing system in England and Wales:

- the introduction of combination orders

- the introduction of the 'unit fine scheme' at magistrates' courts

- the abolition of the sentence of detention in a young offender institution for 14-year-old boys and to a change in the minimum and maximum lengths of sentence to 10 and 12 months respectively to which 15 to 17 -year-olds might be subjected, and

- the abolition of partly suspended sentences of imprisonment and to a restriction in the use of fully suspended sentences

The Criminal Justice Act 1993 abolished the unit fine scheme in magistrates' courts, which had been introduced under the Criminal Justice Act 1991.

A charging standard for assault was introduced in England and Wales on 31 August 1994, the aim being to promote consistency between the police and prosecution on the appropriate level of charge to be brought.

The Criminal Justice and Public Order Act 1994 created several new offences in England and Wales, mainly in the area of public order, but also including male rape (there is no statutory offence of male rape in Scotland, although such a crime may be charged as serious assault). The Act also:

- extended the provisions of section 53 of the Children and Young Persons Act 1993 to 10 to 13-year-olds

- increased the maximum sentence length for 15 to 17 -yearolds to 2 years

- increased the upper limit from $£ 2,000$ to $£ 5,000$ for offences of criminal damage proceeded against as if triable only summarily
- introduced provisions for the reduction of sentences for early guilty pleas and

- increased the maximum sentence length for certain firearm offences

Provisions within the Crime (Sentences) Act 1997 (as amended by the Powers of Criminal Courts Sentencing Act 2000) in England and Wales, and the Crime and Punishment (Scotland) Act 1997 allow for:

- an automatic life sentence for a second serious violent or sexual offence unless exceptional circumstances exist (this provision has not been enacted in Scotland)

- a minimum sentence of 7 years for an offender convicted for a third time of a 'class A' drug trafficking offence unless the court considers this to be unjust in all the circumstances, and

- in England and Wales, section 38A of the Magistrates Courts' Act 1980 (which extends the circumstances in which a magistrates' court may commit a person convicted of an offence triable-either-way to the Crown Court for sentence) was implemented in conjunction with section 49 of the Criminal Procedure and Investigations Act 1996 (when the magistrates' court requires a defendant to indicate a plea before a decision can be taken on the mode of trial and which compels the court to sentence or commit for sentence any defendant who indicates a guilty plea)

Under the Criminal Justice and Court Service Act 2000 new terms were introduced for certain orders. 'Community rehabilitation order' is the new name given to a probation order. A community service order is now known as a 'community punishment order'. Finally, the new term for a combination order is 'community punishment and rehabilitation order'. In April 2000 the secure training order was replaced by the 'detention and training order'. Section 53 of the Children and Young Persons Act 1993 was repealed on 25 August 2000 and its provisions re-enacted in sections 90 to 92 of the Powers of Criminal Courts (Sentencing) Act 2000. 'Reparation and action plan orders' were implemented nationally from 1 June 2000. 'Drug treatment and testing orders' were introduced in England, Scotland and Wales with effect from October 2000. 'Referral orders' were introduced in England, Scotland and Wales with effect from April 2000. These changes are now reflected in Table 11.8.

Following the introduction of the Libra case management system during 2008, offenders at magistrates' courts can now be recorded as sex 'Not Stated'. In 2008 one per cent of offenders sentenced were recorded as sex 'Not Stated'. Amendments to the data tables have been made to accommodate this new category. 


\section{Crime and Justice}

The system of magistrates' courts and Crown Courts in Northern Ireland operates in a similar way to that in England and Wales. A particularly significant statutory development, however, has been the Criminal Justice (NI) Order 1996, which introduces a new sentencing regime to Northern Ireland and largely replicates that introduced to England and Wales by the Criminal Justice Acts of 1991 and 1993. The order makes many changes to both community and custodial sentences and introduces new orders such as the combination order, the custody probation order, and orders for release on licence of sexual offenders.

\section{Expenditure on penal establishments in Scotland}

\section{(Table 11.19)}

The results shown in this table are reported on a cash basis for financial years 1996/97 to 2000/01 in line with funding arrangements. Financial year 2001/02 is reported on a resource accounting basis in line with the introduction of resource budgeting. Capital charges were introduced with resource accounting and budgeting. 
Crime and justice

Numbers

\begin{tabular}{|c|c|c|c|c|c|c|c|c|c|c|c|c|}
\hline & & 1999 & 2000 & 2001 & 2002 & 2003 & 2004 & 2005 & 2006 & 2007 & 2008 & 2009 \\
\hline \multicolumn{13}{|c|}{ England and Wales } \\
\hline \multicolumn{13}{|c|}{ Regular Police(FTE) } \\
\hline \multicolumn{13}{|c|}{ Strength: } \\
\hline Men & KERB & 103956 & 101801 & 102139 & 104483 & 106996 & 110150 & 110597 & 109327 & 108118 & 106866 & 106996 \\
\hline Women & KERC & 19885 & 20155 & 21174 & 22784 & 24430 & 26956 & 28898 & 30307 & 31914 & 32861 & 34651 \\
\hline \multicolumn{13}{|l|}{ Seconded:2,3 } \\
\hline Men & KERD & 2017 & 1965 & 1914 & 2031 & 1689 & 1811 & 1514 & 1545 & 422 & 432 & 438 \\
\hline Women & KERE & 238 & 249 & 292 & 305 & 251 & 284 & 222 & 203 & 60 & 70 & 66 \\
\hline \multicolumn{13}{|c|}{ Additional Officers: ${ }^{4}$} \\
\hline Men & KERF & 324 & 361 & 493 & 567 & 375 & 394 & 522 & 676 & 657 & 661 & 653 \\
\hline Women & KERG & 582 & 519 & 509 & 564 & 709 & 969 & 1042 & 1213 & 1203 & 1471 & 1470 \\
\hline \multicolumn{13}{|c|}{ Special constables } \\
\hline \multicolumn{13}{|c|}{ Strength: 5} \\
\hline Men & KERH & 10860 & 9623 & 8630 & 8014 & 7718 & 7645 & 8074 & 8829 & 9327 & 9719 & 9544 \\
\hline Women & KERI & 5624 & 4724 & 4108 & 3584 & 3319 & 3343 & 3844 & 4350 & 4694 & 4828 & 4707 \\
\hline \multicolumn{13}{|l|}{ Scotland } \\
\hline \multicolumn{13}{|l|}{ Regular police } \\
\hline \multicolumn{13}{|l|}{ Strength: 6} \\
\hline \multicolumn{13}{|c|}{ Central service: ${ }^{7}$} \\
\hline Men & KERM & 88 & 95 & 87 & 116 & 131 & 166 & 195 & 171 & 153 & 219 & 180 \\
\hline Women & KERN & 9 & 13 & 10 & 12 & 17 & 29 & 29 & 21 & 28 & 44 & 37 \\
\hline \multicolumn{13}{|l|}{ Seconded: ${ }^{8}$} \\
\hline Men & KERO & 85 & 130 & 140 & 133 & 166 & 192 & 216 & 200 & 195 & 196 & 291 \\
\hline Women & KERP & 12 & 18 & 14 & 18 & 24 & 30 & 31 & 27 & 28 & 30 & 69 \\
\hline \multicolumn{13}{|c|}{ Additional regular police: } \\
\hline Men & HFVM & 85 & 80 & 83 & 80 & 79 & 88 & 79 & 85 & 107 & 106 & 129 \\
\hline Women & HFVN & 6 & 4 & 5 & 12 & 10 & 13 & 21 & 15 & 12 & 14 & 24 \\
\hline \multicolumn{13}{|c|}{ Special constables } \\
\hline \multicolumn{13}{|l|}{ Strength: } \\
\hline Men & KERS & 1229 & 981 & 924 & 812 & 711 & 773 & 718 & 888 & 886 & 884 & .. \\
\hline Women & KERT & 422 & 355 & 336 & 307 & 280 & 328 & 437 & 432 & 471 & 510 & .. \\
\hline \multirow{2}{*}{\multicolumn{13}{|c|}{$\begin{array}{l}\text { Northern Ireland } \\
\text { Regular police }{ }^{9,10}\end{array}$}} \\
\hline & & & & & & & & & & & & \\
\hline \multicolumn{13}{|l|}{ Strength: } \\
\hline Men & KERU & 7406 & 6844 & 6227 & 6057 & 6171 & 6108 & 6016 & 5992 & 5949 & 5761 & 5669 \\
\hline Women & KERV & 987 & 966 & 1009 & 1080 & 1266 & 1418 & 1547 & 1534 & 1600 & 1653 & 1735 \\
\hline \multicolumn{13}{|l|}{ Reserve $^{11}$} \\
\hline Women & KERX & 641 & 607 & 556 & 510 & 453 & 485 & 410 & 402 & 400 & 382 & 345 \\
\hline
\end{tabular}

1 Figures for England and Wales are as 31 March and are based on full-time 6 'Strength' is WTE police strength, only excluding special constables.

equivalent strength excluding those on career breaks or maternity/paterntiy 7 Instructors at Training Establishments, etc, formerly shown as secondments.

leave. Figures for Scotland are as 31 March. From 1999, figures for North- 8 Includes Scottish Crime and Drug Enforcement Agency .

ern Ireland reflect the position at the end of the financial year, ie 1999 and 9 Does not include officers on secondment.

2000 figures are as 31 March 2000 and 31 March 2001 respectively. Prior 10 Also includes student officers.

to this figures were as at 31 December.

11 Includes part-time reserve and full-time reserve, FTR -515 as at 31 March

2 Figures exclude secondments outside the police service in England and 2009 (481 males and 34 females). Con PT - 760 as at 31 March 2009 (449

Wales (eg to the private sector or to law enforcement agencies overseas). males and 311 females). FTR -382 as at 31 March 2010 (362 males and 20

3 From 31 March 2007 onwards details of officers seconded to NCIS and females) Con PT -703 as at 31 March 2010 (412 males and 291 female)

NCS will no longer appear following the launch of Serious Organised Crime Agency (SOCA) in April 2006.

4 Figures include those officers on career breaks or maternity/paternity leave.

Sources: Home Office: 0207035 0289;

Prior to 2003, these figures were not collected centrally.

Scottish Government $U$

5 Special constable figures are given as a headcount measure. The Police Service of Northern Ireland: 08456008000 ext 24070 


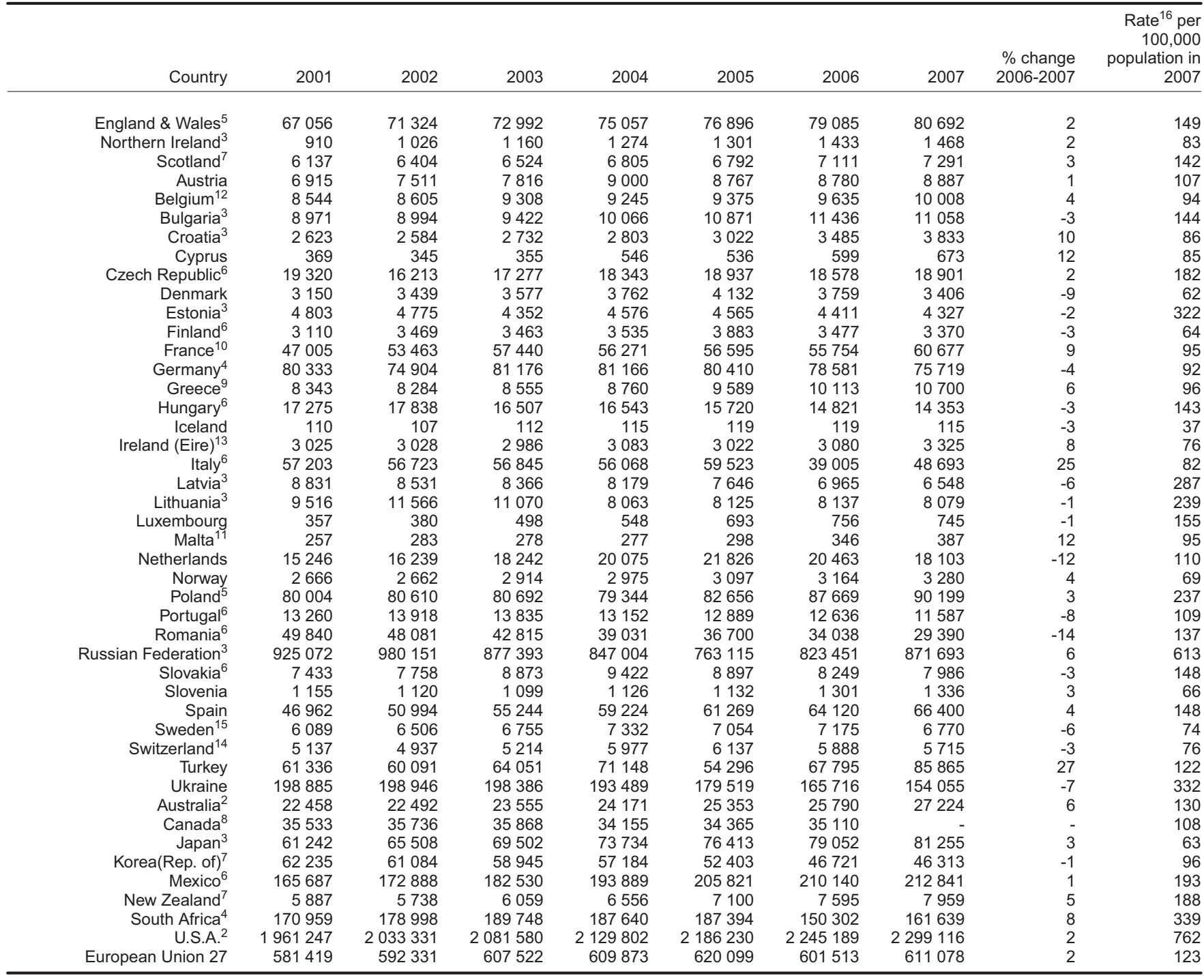

1 At 1 September: number of prisoners, including pre-trial detainees/remand

Sources: Ministries responsible for prisons, national prison administrations; national statistical offices, Council of Europe Annual Penal Statistics (SPACE) prisoners.

2 At 30 June.

3 At 1 January. World Prison Population List and World Prison Brief;

4 At 31 March.

5 At 31 August.

6 At 31 December.

7 Annual averages. Countries calculate these on the basis of daily, weekly or monthly figures.

8 Annual averages by financial year (e.g. 2006=1 April 2005-31 March 2006).

Rate per 100,000 population reflects the position in 2006

9 At 1 September (2001-03, 2005-06). At 16 December (2004). At 30 June (2007).

10 Metropolitan and overseas departments and territories.

11 At 1 September (2001-06). Annual average (2007).

12 At 1 March.

13 At 1 September (2001-06). At 26 October (2007).

14 At third Wednesday in March (2001). At first Wednesday in September (2002-07).

15 At 1 October.

16 Based on estimates of national population. 
Crime and justice

\section{Recorded crime statistics: by offence group ${ }^{1}$ \\ England and Wales}

Thousands

\begin{tabular}{|c|c|c|c|c|c|c|c|c|c|c|c|c|c|c|}
\hline & & 1997 & $1998^{2}$ & & $\begin{array}{r}1999 \\
/ 00\end{array}$ & $\begin{array}{r}2000 \\
/ 01\end{array}$ & $\begin{array}{c}2001^{4} \\
/ 02\end{array}$ & $\begin{array}{r}2002^{4} \\
103\end{array}$ & $\begin{array}{r}2003 \\
104\end{array}$ & $\begin{array}{r}2004 \\
105\end{array}$ & $\begin{array}{r}2005 \\
106\end{array}$ & $\begin{array}{c}2006^{8} \\
107\end{array}$ & $\begin{array}{r}8007 \\
108\end{array}$ & $\begin{array}{r}2008 \\
109\end{array}$ \\
\hline Violence against the person & BEAB & 250.8 & 230.8 & LQMP & 581.0 & 600.9 & 650.3 & 845.1 & 967.2 & 1048.1 & 1059.6 & 1046.4 & 961.1 & 903.4 \\
\hline Sexual offences ${ }^{7}$ & BEAC & 33.2 & 34.9 & LQMQ & 37.8 & 37.3 & 41.4 & 58.9 & 62.5 & 62.9 & 62.1 & 57.5 & 53.5 & 51.4 \\
\hline Burglary & BEAD & 1015.1 & 951.9 & LQMR & 906.5 & 836.0 & 878.5 & 890.1 & 820.0 & 680.4 & 645.1 & 622.0 & 583.7 & 581.5 \\
\hline Robbery & BEAE & 63.1 & 66.2 & LQMS & 84.3 & 95.2 & 121.4 & 110.3 & 103.7 & 91.0 & 98.2 & 101.4 & 84.7 & 80.1 \\
\hline $\begin{array}{l}\text { Theft and handling stolen goods (of which): } \\
\text { Offences against vehicles } \\
\text { Other theft offences }\end{array}$ & BEAF & 2165.0 & 2126.7 & $\begin{array}{l}\text { LQMT } \\
\text { I } 8 \mathrm{RM} \\
\text { I } 8 \mathrm{RN}\end{array}$ & $\begin{array}{r}2223.6 \\
. . \\
.\end{array}$ & $\begin{array}{r}2145.4 \\
\cdots \\
\cdots\end{array}$ & $\begin{array}{r}2267.0 \\
. . \\
.\end{array}$ & $\begin{array}{rr}1 & - \\
1 & 074.7 \\
1 & 336.9\end{array}$ & $\begin{array}{r}-\bar{a} \\
985.0 \\
1327.9\end{array}$ & $\begin{array}{r}-\overline{1} \\
820.1 \\
1247.6\end{array}$ & $\begin{array}{r}792 . \overline{8} \\
1226.2\end{array}$ & $\begin{array}{r}765.0 \\
1180.8\end{array}$ & $\begin{array}{r}656 . \overline{4} \\
1121.0\end{array}$ & $\begin{array}{r}591.9 \\
1080.0\end{array}$ \\
\hline Fraud and forgery & BEAG & 134.4 & 173.7 & LQMU & 334.8 & 319.3 & 314.9 & 331.1 & 319.6 & 280.1 & 232.8 & 199.7 & 155.3 & 163.1 \\
\hline Criminal damage & BEAH & 877.0 & 834.4 & LQMV & 945.7 & 960.1 & 1064.5 & 1120.6 & 1218.5 & 1197.5 & 1184.3 & 1185.0 & 1036.2 & 936.4 \\
\hline Drug offences ${ }^{5}$ & LQMO & .. & 21.3 & LQYT & 121.9 & 113.5 & 121.3 & 143.3 & 143.5 & 145.8 & 178.5 & 194.2 & 229.0 & 243.4 \\
\hline Total & BEAA & 4598.3 & 4481.8 & LQYV & $\overline{5301.2}$ & 5170.8 & 5527.1 & 5975.0 & 6013.8 & 5637.5 & $\overline{5555.2}$ & 5427.6 & 4951.2 & 4702.5 \\
\hline $\begin{array}{l}1 \text { See chapter text. } \\
2 \text { Estimates. } \\
3 \text { Figures from this period are not direc } \\
1998 / 99 \text { and from } 2002 / 03 \text { onwards. } \\
4 \text { The National Crime Recording Standar } \\
\text { land and Wales from } 1 \text { April } 2002 \text {. Thes } \\
\text { ble with those for earlier years. }\end{array}$ & $\begin{array}{l}\text { ctly con } \\
\text { rd (NCF } \\
\text { se figure }\end{array}$ & $\begin{array}{l}\text { parable } \\
\text { S) was in } \\
\text { s are not }\end{array}$ & $\begin{array}{l}\text { with date } \\
\text { ntroduce } \\
\text { directly }\end{array}$ & $\begin{array}{l}\text { a prior } \\
\text { d in En } \\
\text { compar }\end{array}$ & $\begin{array}{rr}\text { to } & \text { ing } \\
& \text { se } \\
\text { ig- } 6 \text { Inc } \\
\text { ra- } 7 \text { Th } \\
\text { an } \\
8 \mathrm{Th}\end{array}$ & $\begin{array}{l}\text { ior to } 1 \\
\text { fences' } \mathrm{g} \\
\mathrm{g} \text { becam } \\
\text { ession an } \\
\text { cludes th } \\
\text { he Sexua } \\
\text { id covera }\end{array}$ & $\begin{array}{l}\text { April } 199 \\
\text { group. Fro } \\
\text { le part of } \\
\text { רd other d } \\
\text { he British } \\
\text { al Offence } \\
\text { age of sex }\end{array}$ & $\begin{array}{l}98 \text { the off } \\
\text { om } 1 \text { Apri } \\
\text { f a new 'D } \\
\text { drug offen } \\
\text { Transpor } \\
\text { es Act } 20 \\
\text { xual offen }\end{array}$ & $\begin{array}{l}\text { fence of c } \\
\text { ril } 1999, \mathrm{u} \\
\text { Drug offen } \\
\text { nces. } \\
\text { rt Police ( } \\
\text { 003, introc } \\
\text { nces. }\end{array}$ & $\begin{array}{l}\text { drug traff } \\
\text { under the } \\
\text { nces' gro } \\
\text { (BTP) fro } \\
\text { duced in }\end{array}$ & $\begin{array}{l}\text { fficking w } \\
\text { e new col } \\
\text { oup which } \\
\text { om 2002/ } \\
\text { n May } 200 \\
07 \text { and ba }\end{array}$ & $\begin{array}{l}\text { vas includ } \\
\text { unting rul } \\
\text { h, now als } \\
\text { /03 onwar } \\
04 \text {, altere }\end{array}$ & $\begin{array}{l}\text { ded in th } \\
\text { les, drug } \\
\text { lso includ } \\
\text { ards. } \\
\text { ed the de } \\
\text { to } 2002 \text { / }\end{array}$ & $\begin{array}{l}\text { e 'Other } \\
\text { traffick- } \\
\text { des pos- } \\
\text { efinitions } \\
\text { /03. }\end{array}$ \\
\hline
\end{tabular}




\begin{tabular}{|c|c|c|c|c|c|c|c|c|c|c|c|c|}
\hline & & 1998 & 1999 & 2000 & 2001 & 2002 & 2003 & 2004 & 2005 & 2006 & 2007 & $2008^{6}$ \\
\hline \multicolumn{13}{|l|}{ All ages ${ }^{4}$} \\
\hline \multicolumn{13}{|l|}{ Indictable offences } \\
\hline Violence against the person: & KJEJ & 37.1 & 35.7 & 35.3 & 35.3 & 37.7 & 38.0 & 39.1 & 40.9 & 41.9 & 42.0 & 41.5 \\
\hline Murder & KESB & 0.3 & 0.3 & 0.3 & 0.3 & 0.3 & 0.3 & 0.4 & 0.4 & 0.4 & 0.4 & 0.4 \\
\hline Manslaughter & KESC & 0.3 & 0.2 & 0.2 & 0.3 & 0.3 & 0.2 & 0.3 & 0.3 & 0.2 & 0.2 & 0.2 \\
\hline Wounding & KESD & 35.2 & 33.9 & 33.5 & 33.5 & 35.7 & 35.9 & 36.9 & 38.6 & 39.8 & 39.8 & 39.3 \\
\hline against the person & KESE & 1.3 & 1.4 & 1.3 & 1.2 & 1.4 & 1.6 & 1.6 & 1.6 & 1.5 & 1.5 & 1.5 \\
\hline Sexual offences & KESF & 4.6 & 4.3 & 3.9 & 4.0 & 4.4 & 4.4 & 4.8 & 4.8 & 4.9 & 5.1 & 5.1 \\
\hline Burglary & KESG & 30.8 & 29.3 & 26.2 & 24.8 & 26.7 & 25.7 & 24.3 & 23.0 & 23.0 & 23.8 & 23.9 \\
\hline Robbery & KESH & 5.5 & 5.6 & 5.9 & 6.8 & 7.7 & 7.3 & 7.5 & 7.1 & 8.1 & 8.8 & 8.5 \\
\hline Theft and handling stolen goods & KESI & 125.7 & 131.2 & 128.0 & 127.0 & 127.3 & 119.1 & 110.6 & 103.8 & 99.0 & 106.0 & 110.9 \\
\hline Fraud and forgery & KESJ & 19.8 & 20.3 & 19.2 & 18.3 & 18.1 & 18.0 & 18.1 & 18.5 & 18.2 & 19.9 & 19.8 \\
\hline Criminal damage & KESK & 10.9 & 10.9 & 10.3 & 10.7 & 11.0 & 11.2 & 11.7 & 11.7 & 12.7 & 12.5 & 9.6 \\
\hline Drug offences & KBWX & 48.8 & 48.7 & 44.6 & 45.6 & 49.0 & 51.2 & 39.2 & 39.1 & 39.6 & 44.6 & 52.9 \\
\hline \multicolumn{13}{|l|}{ Other offences (excluding } \\
\hline Total & KESA & 341.7 & 342.0 & 325.5 & 324.2 & 338.3 & 335.1 & 317.8 & 308.5 & 303.2 & 313.3 & 316.9 \\
\hline \multicolumn{13}{|l|}{ Summary offences } \\
\hline Summary assaults & KESO & 35.3 & 37.5 & 37.4 & 37.7 & 40.7 & 45.6 & 53.4 & 60.4 & 64.5 & 68.9 & 67.7 \\
\hline Offences against Public Order & JW94 & 31.3 & 30.8 & 29.8 & 28.6 & 29.0 & 31.0 & 33.5 & 32.6 & 34.9 & 38.7 & 37.0 \\
\hline Firearms Acts & JW95 & 0.5 & 0.4 & 0.4 & 0.3 & 0.3 & 0.3 & 0.5 & 0.8 & 0.6 & 0.7 & 0.5 \\
\hline Interference with a motor vehicle & JW96 & 2.7 & 2.8 & 2.6 & 2.6 & 2.6 & 2.6 & 2.5 & 2.4 & 2.3 & 2.3 & 1.9 \\
\hline \multicolumn{13}{|l|}{ Stealing or unauthorised } \\
\hline taking of a conveyance & JW97 & 6.9 & 7.5 & 7.0 & 6.9 & 7.2 & 6.6 & 5.9 & 5.2 & 4.8 & 4.6 & 3.9 \\
\hline Social Security Offences & JW98 & 5.9 & 5.0 & 6.5 & 7.5 & 6.8 & 7.0 & 6.9 & 6.3 & 4.0 & 3.3 & 3.4 \\
\hline \multicolumn{13}{|l|}{ Intoxicating Liquor Laws: } \\
\hline Drunkenness & KESR & 30.8 & 28.7 & 27.2 & 26.2 & 26.9 & 27.7 & 21.1 & 16.1 & 15.7 & 17.4 & 18.9 \\
\hline Education Acts & KEST & 5.0 & 5.1 & 5.1 & 5.6 & 5.8 & 5.8 & 6.5 & 6.4 & 7.4 & 8.4 & 8.5 \\
\hline \multicolumn{13}{|l|}{ Summary offences of criminal } \\
\hline Criminal Damage - $£ 5,000$ or less & KESW & 26.5 & 27.9 & 28.0 & 26.9 & 28.3 & 29.8 & 31.5 & 31.1 & 30.2 & 32.1 & 33.3 \\
\hline Offences by prostitutes & KESX & 5.2 & 3.4 & 3.4 & 2.8 & 2.7 & 2.6 & 1.7 & 1.1 & 0.7 & 0.5 & 0.5 \\
\hline TV licence evasion & KETC & 76.6 & 55.8 & 105.7 & 83.8 & 96.6 & 79.9 & 89.3 & 105.0 & 115.5 & 121.0 & 122.0 \\
\hline Motoring offences (summary) & KETA & 665.2 & 632.9 & 607.5 & 583.3 & 595.8 & 662.6 & 707.9 & 667.1 & 622.5 & 611.1 & 552.2 \\
\hline
\end{tabular}

Persons aged 10 to under $18^{5}$

Indictable offences

Wounding

Other offences of violence

against the person

Sexual offences

Burglary

Robbery

Theft and handling stolen goods

Fraud and forgery

Criminal damage

Drug offences

Other offences (excluding

motoring)

Motoring

Total

KETF
KBXC
KCAA
KETG
KETH
KETI
KETJ
KETK
KETL
KCAB
KETM
KETN
KETE

$\begin{array}{rrr}6.0 & 6.0 \\ 5.9 & 5.9 \\ & & \\ 0.1 & 0.1 & \\ 0.5 & 0.5 & \\ 8.5 & 7.8 & \\ 2.2 & 2.0 & \\ 21.9 & 22.7 & 2 \\ 1.0 & 1.1 & \\ 2.3 & 2.7 & \\ 2.7 & 3.1 & \\ & & \\ 4.2 & 4.3 & \\ 0.4 & 0.4 & \\ 49.7 & 50.6 & 4.4 \\ & \end{array}$

$\begin{array}{rrrr}6.4 & 6.9 & 6.9 & 6.6 \\ 6.3 & 6.8 & 6.8 & 6.5 \\ 0.1 & 0.1 & 0.1 & 0.1 \\ 0.5 & 0.5 & 0.6 & 0.4 \\ 6.8 & 6.3 & 6.4 & 5.8 \\ 2.2 & 2.8 & 2.8 & 2.6 \\ 21.0 & 20.6 & 18.4 & 16.5 \\ 1.0 & 1.0 & 0.9 & 0.8 \\ 2.6 & 2.9 & 2.9 & 2.9 \\ 3.7 & 4.3 & 5.0 & 5.1 \\ & & & \\ 4.4 & 4.3 & 4.4 & 4.3 \\ 0.6 & 0.7 & 0.8 & 0.8 \\ 49.2 & 50.3 & 49.1 & 46.0 \\ & & & \end{array}$

$\begin{array}{rr}6.6 & 6.9 \\ 6.5 & 6.8 \\ & \\ 0.1 & 0.1 \\ 0.4 & 0.6 \\ 5.8 & 5.9 \\ 2.6 & 3.0 \\ 16.5 & 16.8 \\ 0.8 & 0.8 \\ 2.9 & 3.2 \\ 5.1 & 4.5 \\ & \\ 4.3 & 4 . \\ 0.8 & 0.7 \\ 46.0 & 47.0\end{array}$

$\begin{array}{rr}6.9 & 7.4 \\ 6.8 & 7.3 \\ 0.1 & 0.1 \\ 0.6 & 0.6 \\ 5.9 & 6.0 \\ 3.0 & 3.1 \\ 16.8 & 17.1 \\ 0.8 & 0.7 \\ 3.2 & 3.3 \\ 4.5 & 4.6 \\ 4.6 & \\ 0.7 & \\ 47.0 & 47.8\end{array}$

$\begin{array}{rrrr}7.4 & 7.5 & 7.7 & 7.4 \\ 7.3 & 7.4 & 7.6 & 7.3 \\ & & & \\ 0.1 & 0.1 & 0.1 & 0.1 \\ 0.6 & 0.5 & 0.5 & 0.5 \\ 6.0 & 6.2 & 6.1 & 5.4 \\ 3.1 & 3.7 & 4.1 & 3.6 \\ 17.1 & 16.3 & 18.2 & 16.0 \\ 0.7 & 0.6 & 0.7 & 0.5 \\ 3.3 & 3.7 & 3.6 & 2.6 \\ 4.6 & 4.5 & 5.3 & 6.4 \\ & & & \\ 4.5 & 4.1 & 4.2 & 3.5 \\ 0.6 & 0.5 & 0.4 & 0.3 \\ 47.8 & 47.6 & 50.9 & 46.3\end{array}$

\section{Summary offences}

Summary assaults

Offences against Public Order

Firearms Acts

TW9

$\begin{array}{lll}5.0 & 5.8 \quad 6.3\end{array}$

Interference with a motor vehicle

JW9A

JW9B

3.9
0.1
0.9

$6.3 \quad 6.8$

$7.3 \quad 7.9$

$9.5 \quad 11$

Stealing or unauthorised

taking of a conveyance

Criminal Damage $-£ 5,000$ or less

Intoxicating liquor laws

Drunkenness

Motoring offences (summary) $\quad$ JW9E

Other summary non-motoring offences JX3D

Total

KETO

3.0

1 See chapter text.

4 Includes 'Companies', etc .

2 Data provided on the principal offence basis

5 Figures for persons aged 10 to under 18 are included in the totals above.

3 Every effort is made to ensure that the figures presented are accurate and complete. However, it is important to note that these data have been extracted from large administrative data systems generated by the courts and police forces. As a consequence, care should be taken to ensure data collection processes and their inevitable limitations are taken into account 2008 figures exclude data for Cardiff magistrates' court for April, July and August 2008. 
Thousands

\begin{tabular}{|c|c|c|c|c|c|c|c|c|c|c|c|c|}
\hline & & 1998 & 1999 & 2000 & 2001 & 2002 & 2003 & 2004 & 2005 & 2006 & 2007 & 2008 \\
\hline \multicolumn{13}{|l|}{ All ages ${ }^{4}$} \\
\hline \multicolumn{13}{|l|}{ Indictable offences } \\
\hline Violence against the person: & KELB & 23.5 & 21.2 & 19.9 & 19.6 & 23.6 & 28.8 & 36.6 & 51.0 & 57.3 & 52.3 & 37.6 \\
\hline Wounding & KCAF & 22.9 & 20.6 & 19.3 & 18.9 & 22.9 & 27.9 & 35.4 & 49.6 & 55.7 & 50.8 & 36.1 \\
\hline Other violence against the person & KCAG & 0.6 & 0.6 & 0.6 & 0.6 & 0.7 & 0.9 & 1.2 & 1.4 & 1.5 & 1.6 & 1.6 \\
\hline Sexual offences & KELC & 1.7 & 1.5 & 1.3 & 1.2 & 1.2 & 1.4 & 1.6 & 1.8 & 1.9 & 2.0 & 1.7 \\
\hline Robbery & KELE & 0.6 & 0.6 & 0.6 & 0.5 & 0.4 & 0.4 & 0.5 & 0.6 & 0.7 & 0.6 & 0.4 \\
\hline Theft and handling stolen goods & KELF & 83.6 & 75.4 & 67.6 & 63.5 & 54.2 & 54.5 & 61.9 & 67.6 & 72.4 & 72.8 & 63.8 \\
\hline Fraud and forgery & KELG & 7.4 & 7.2 & 6.2 & 5.8 & 5.3 & 5.5 & 6.0 & 6.9 & 8.0 & 8.6 & 8.2 \\
\hline Criminal damage & KELH & 2.7 & 3.0 & 3.2 & 3.4 & 3.1 & 3.7 & 5.5 & 7.2 & 9.0 & 8.8 & 7.7 \\
\hline Drug offences & KCAI & 58.7 & 49.4 & 41.1 & 39.4 & 44.9 & 45.7 & 32.6 & 34.4 & 37.4 & 43.1 & 46.9 \\
\hline Other offences & KELI & 5.0 & 4.6 & 4.4 & 4.1 & 4.4 & 5.3 & 6.0 & 6.9 & 9.4 & 10.0 & 8.6 \\
\hline Total & KELA & 191.7 & 170.6 & 150.9 & 143.9 & 142.9 & 150.7 & 156.3 & 182.9 & 203.8 & 205.1 & 180.3 \\
\hline Summary assaults & KELK & 13.2 & 17.0 & 17.2 & 18.2 & 17.3 & 19.8 & 26.1 & 40.8 & 64.6 & 72.6 & 69.5 \\
\hline Offences against Public Order & JW9W & 14.5 & 14.6 & 13.4 & 13.0 & 12.7 & 15.1 & 15.4 & 15.3 & 18.5 & 20.6 & 19.0 \\
\hline Firearms Acts & JW9x & 0.9 & 0.7 & 0.6 & 0.4 & 0.3 & 0.2 & 0.8 & 1.1 & 1.0 & 1.1 & 0.6 \\
\hline Interference with a motor vehicle & JW9Y & 0.5 & 0.6 & 0.5 & 0.5 & 0.5 & 0.5 & 0.4 & 0.5 & 0.6 & 0.6 & 0.4 \\
\hline Stealing or unauthorised & & & & & & & & & & & & \\
\hline taking of a conveyance & JW9z & 4.5 & 4.5 & 4.3 & 4.4 & 3.9 & 3.7 & 3.3 & 3.2 & 3.3 & 3.2 & 2.6 \\
\hline Social Security Offences & JWA2 & 0.1 & 0.1 & 0.2 & 0.2 & 0.5 & 0.4 & 0.4 & 0.5 & 0.5 & 0.6 & 0.7 \\
\hline Intoxicating Liquor Laws: & & & & & & & & & & & & \\
\hline Drunkenness & KELN & 47.0 & 45.9 & 42.4 & 41.7 & 39.7 & 44.4 & 45.8 & 45.8 & 48.3 & 49.8 & 44.4 \\
\hline Education Acts & KELP & - & - & - & - & - & 0.1 & 0.1 & 0.1 & 0.1 & 0.1 & 0.1 \\
\hline Offences by prostitutes & KELT & 0.1 & 0.1 & - & - & - & - & - & - & - & - & - \\
\hline TV licence evasion & KELY & 6.4 & 5.7 & 5.0 & 4.5 & 4.2 & 4.0 & 4.5 & 6.3 & 7.1 & 7.3 & 7.3 \\
\hline Other summary non-motoring offences & $\mathrm{JX} 6 \mathrm{~L}$ & 9.1 & 6.4 & 4.5 & 3.0 & 3.1 & 3.0 & 2.8 & 2.4 & 2.2 & 2.1 & 2.2 \\
\hline Total & KELU & 96.2 & 95.6 & 88.1 & 85.9 & 82.4 & 91.1 & 99.5 & 116.0 & 146.2 & 157.8 & 146.6 \\
\hline
\end{tabular}

Persons aged 10 to under $18^{5}$

Indictable offences

Other violence against the

person

Sexual offences

Burglary

Robbery

Theft and handling stolen goods

Fraud and forgery

Criminal damage

Drug offences

Other offences

Total

Summary offences

Summary assaults

Offences against Public Order

Firearms Acts

Interference with a motor vehicle

Stealing or unauthorised

taking of a conveyance

Criminal Damage - $£ 5,000$ or less

Intoxicating liquor laws:

Drunkenness

Other summary non-motoring offences

KEMB
KCAP
KCCE
KEMC
KEMD
KEME
KEMF
KEMG
KEMH
KCCF
KEMI

\begin{tabular}{|c|c|}
\hline 9.5 & 8.5 \\
\hline 9.4 & 8.4 \\
\hline - & 0.1 \\
\hline 0.6 & 0.6 \\
\hline 6.7 & 6.1 \\
\hline 0.5 & 0.5 \\
\hline 44.0 & 39.6 \\
\hline 1.6 & 1.7 \\
\hline 1.7 & 1.9 \\
\hline 11.0 & 9.6 \\
\hline 1.5 & 1.4 \\
\hline & 69.8 \\
\hline
\end{tabular}

8.3

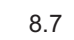

$9.3 \quad 11$

$13.0-13$.

0.1

$\begin{array}{lll}0.1 & 0.1 \\ 0.4 & 0.5 & \end{array}$

$\begin{array}{ll}5.4 & 5.3 \\ 0.5 & 0.5\end{array}$

$36.9 \quad 35.2$

$1.5 \quad 1.3$

$2.1 \quad 2.3$

$\begin{array}{ll}7.9 & 8.5 \\ 1.3 & 1.3\end{array}$

$1.3 \quad 1.3$

KEMA

64.3

63.5

\section{1}

0.4
4.6

4.6
0.4

$28.1 \quad 28.3$

1.1

1.9

$9.5 \quad 9.6$

1.31 .4

$\overline{56.6} \quad \overline{58.7}$

$0.1-0.1$

$\begin{array}{llll}16.5 & 16.6 & 13.9 & 9.3\end{array}$

$\begin{array}{llll}16.4 & 16.5 & 13.8 & 9.3\end{array}$

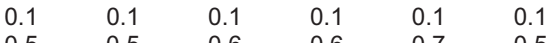

$\begin{array}{llllll}0.4 & 4.2 & 4.6 & 0.6 & 0.7 & 0.5 \\ 0.4 & 0.4 & 0.5 & 0.6 & 4.5 & 3.2\end{array}$

\begin{tabular}{rrrrrr}
0.4 & 0.4 & 0.5 & 0.6 & 0.5 & 0.3 \\
\hline 8.3 & 33.1 & 36.8 & 39.4 & 39.7 & 30.0
\end{tabular}

$\begin{array}{rrrrrr}1.0 & 1.0 & 1.1 & 1.3 & 1.4 & 30.0\end{array}$

$\begin{array}{llllll}1.0 & 1.0 & 1.1 & 1.3 & 1.4 & 1.1 \\ 2.3 & 3.1 & 3.9 & 4.7 & 4.5 & 3.5\end{array}$

$\begin{array}{llllll}.6 & 8.3 & 7.8 & 7.1 & 8.1 & 8.5\end{array}$

\begin{tabular}{lllll}
1.6 & 1.6 & 1.9 & 2.0 & 1.6 \\
\hline
\end{tabular}

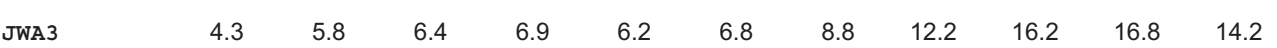

$\begin{array}{rrrrrrrrrrrr}\text { JWA3 } & 4.3 & 5.8 & 6.4 & 6.9 & 6.2 & 6.8 & 8.8 & 12.2 & 16.2 & 16.8 & 14.2 \\ \text { JWA5 } & 4.2 & 4.3 & 4.0 & 4.1 & 3.8 & 4.6 & 5.8 & 6.2 & 7.4 & 7.3 & 5.8 \\ \text { JWA4 } & 0.6 & 0.5 & 0.4 & 0.3 & 0.2 & 0.1 & 0.4 & 0.6 & 0.5 & 0.6 & 0.3\end{array}$

JWA4

JWA6

$\begin{array}{llll}0.6 & 0.5 & 0.4 & 0.3 \\ 0.3 & 0.4 & 0.4 & 0.4\end{array}$

$\begin{array}{ll}0.2 & 0.1 \\ 0.4 & 0.4\end{array}$

0.4

0.3

$0.3 \quad 0.3$

0.3

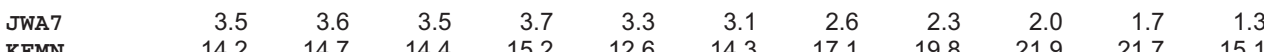

KEMN

$\begin{array}{rr}3.5 & 3.6 \\ 14.2 & 14.7\end{array}$

JWA8

$\begin{array}{lllllllllll}2.9 & 2.6 & 2.3 & 2.3 & 2.1 & 2.4 & 2.5 & 1.9 & 1.5 & 1.6 & 1.5\end{array}$

JX6M

$\frac{2.5}{32.5} \quad \frac{1.9}{34.2}$

KEMJ

32.5

33.2

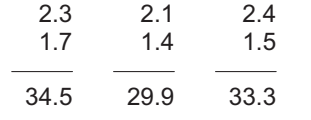

\begin{tabular}{|c|c|c|c|}
\hline 2.5 & 1.9 & 1.5 & 1.6 \\
\hline 1.6 & 2.1 & 2.1 & 2.2 \\
\hline 9.1 & 45.5 & 52.0 & 52.1 \\
\hline
\end{tabular}

1 See chapter text.

2 Data provided on the principal offence basis.

3 Every effort is made to ensure that the figures presented are accurate and

forces. As a consequence, care should be taken to ensure data collection processes and their inevitable limitations are taken into account when those data complete. However, it is important to note that these data have been exare used.

tracted from large administrative data systems generated by police

4 Includes 'Companies', etc.

5 Figures for persons aged 10 to under 18 are included in the totals above.

Source: Justice Statistics Analytical Services in the Ministry of Justice 020 


\begin{tabular}{|c|c|c|c|c|c|c|c|c|c|c|c|c|c|}
\hline & & & 1998 & 1999 & 2000 & 2001 & 2002 & 2003 & 2004 & 2005 & 2006 & 2007 & 2008 \\
\hline \multicolumn{14}{|l|}{ Males } \\
\hline \multicolumn{14}{|l|}{ Indictable offences } \\
\hline All ages & $\mathbf{K}$ & $\mathbf{A}$ & 292.9 & 291.7 & 276.5 & 275.5 & 287.1 & 283.4 & 268.4 & 261.3 & 258.4 & 266.9 & 267.7 \\
\hline 10 and under 15 years & $\mathbf{K}$ & B & 8.1 & 8.9 & 8.7 & 9.0 & 8.8 & 8.0 & 8.5 & 8.6 & 8.3 & 8.5 & 7 \\
\hline 15 and under 18 years & $\mathbf{K}$ & C & 35.2 & 35.1 & 33.8 & 34.4 & 33.7 & 31.4 & 31.8 & 32.0 & 32.5 & 35.0 & 31.9 \\
\hline 18 and under 21 years & $\mathbf{K}$ & & 51.8 & 52.6 & 49.9 & 48.2 & 46.6 & 43.8 & 39.9 & 38.5 & 39.0 & 40.6 & 38 \\
\hline 21 years and over & $\mathbf{K}$ & & 197.9 & 195.0 & 184.0 & 183.9 & 198.0 & 200.2 & 188.2 & 182.2 & 178.7 & 182.9 & 189 \\
\hline All ages & $\mathbf{K}$ & & 929.0 & 886.6 & 881.0 & 826.6 & 866.4 & 937.1 & 990.0 & 931.2 & 877.7 & 851.5 & 78 \\
\hline 10 and under 15 years & $\mathbf{K}$ & G & 3.9 & 5.1 & 5.8 & 6.2 & 6.1 & 6.1 & 6.7 & 7.2 & 7.0 & 7.3 & \\
\hline 15 and under 18 years & $\mathbf{K}$ & H & 28.5 & 30.3 & 32.2 & 34.5 & 34.6 & 35.3 & 36.4 & 34.6 & 32.3 & 31.9 & 28 \\
\hline 18 and under 21 years & $\mathbf{K}$ & I & 96.3 & 94.8 & 93.0 & 92.2 & 94.7 & 99.9 & 98.2 & 89.4 & 85.1 & 80.5 & 76 \\
\hline 21 years and over & $\mathbf{K}$ & & 800.3 & 756.5 & 750.0 & 693.6 & 731.0 & 795.8 & 848.8 & 800.1 & 753.4 & 731.8 & 667 \\
\hline
\end{tabular}

\begin{tabular}{|c|c|c|c|c|c|c|c|c|c|c|c|c|c|}
\hline \multicolumn{14}{|l|}{$\begin{array}{l}\text { Females } \\
\text { Indictable offences }\end{array}$} \\
\hline All ages & $\mathbf{K}$ & $\mathbf{K}$ & 47.3 & 49.0 & 47.7 & 47.4 & 50.0 & 50.2 & 48.4 & 46.1 & 43.7 & 45.3 & 46.4 \\
\hline 10 and under 15 years & $\mathbf{K}$ & & 1.4 & 1.4 & 1.5 & 1.6 & 1.6 & 1.6 & 1.7 & 1.7 & 1.7 & 1.8 & 1.6 \\
\hline 15 and under 18 years & $\mathbf{K}$ & M & 5.1 & 5.2 & 5.2 & 5.3 & 5.1 & 4.9 & 5.0 & 5.5 & 5.1 & 5.5 & 4.9 \\
\hline 18 and under 21 years & $\mathbf{K}$ & & 7.1 & 7.6 & 7.5 & 7.0 & 6.9 & 6.2 & 5.7 & 5.3 & 4.8 & 4.6 & 4.7 \\
\hline 21 years and over & $\mathbf{k}$ & o & 33.7 & 34.7 & 33.5 & 33.5 & 36.5 & 37.5 & 35.9 & 33.6 & 32.1 & 33.4 & 35.3 \\
\hline \multicolumn{14}{|l|}{ Summary offences } \\
\hline All ages & $\mathbf{K}$ & $\mathbf{P}$ & 188.3 & 171.0 & 208.3 & 190.2 & 208.7 & 210.5 & 231.2 & 236.6 & 233.9 & 244.2 & 242.0 \\
\hline 10 and under 15 years & $\mathbf{K}$ & $\mathbf{Q}$ & 0.6 & 0.8 & 0.9 & 0.9 & 1.1 & 1.2 & 1.4 & 1.6 & 1.6 & 1.8 & 1.7 \\
\hline 15 and under 18 years & $\mathbf{k}$ & $\mathbf{R}$ & 3.8 & 3.4 & 3.3 & 3.6 & 3.6 & 4.0 & 4.6 & 4.9 & 5.2 & 5.7 & \\
\hline 18 and under 21 years & $\mathbf{K}$ & $\mathbf{s}$ & 12.1 & 10.8 & 11.8 & 11.1 & 11.6 & 12.6 & 13.0 & 13.5 & 14.2 & 14.9 & 16.5 \\
\hline 21 years and over & $\mathbf{K}$ & $\mathbf{T}$ & 171.7 & 155.9 & 192.3 & 174.7 & 192.4 & 192.7 & 212.1 & 216.6 & 212.9 & 221.9 & 218.3 \\
\hline \multicolumn{14}{|l|}{ Companies, etc } \\
\hline Indictable offences & $\mathbf{K}$ & U & 1.5 & 1.3 & 1.3 & 1.3 & 1.2 & 1.4 & 1.1 & 1.1 & 1.0 & 1.1 & \\
\hline Summary offences & $\mathbf{k}$ & $\mathrm{v}$ & 10.7 & 8.9 & 8.8 & 8.6 & 7.9 & 8.6 & 9.4 & 8.1 & 6.6 & 6.9 & 25.8 \\
\hline $\begin{array}{l}1 \text { See chapter text. } \\
2 \text { These data are on the pr } \\
3 \text { Every effort is made to } \\
\text { complete. However, it is } \\
\text { tracted from large admin }\end{array}$ & & & $\begin{array}{l}\text { reser } \\
\text { these }\end{array}$ & $\begin{array}{l}\text { re ac } \\
\text { a hav }\end{array}$ & $\begin{array}{l}e \text { and } \\
\text { en ex- }\end{array}$ & $\begin{array}{l}\text { and } \\
\text { collec } \\
\text { when } \\
4 \text { Exclu }\end{array}$ & $\begin{array}{l}\text { forc } \\
\text { proce } \\
\text { e dat } \\
\text { data } f\end{array}$ & $\begin{array}{l}\text { As a } \\
\text { s and } \\
\text { e used. } \\
\text { ardiff me }\end{array}$ & $\begin{array}{l}\text { eque } \\
r \text { ine } \\
\text { trate }\end{array}$ & $\begin{array}{l}\text { care } \\
\text { le lim }\end{array}$ & be & $\begin{array}{l}\text { to } e \\
\text { en in }\end{array}$ & \\
\hline
\end{tabular}

Thousands

\begin{tabular}{|c|c|c|c|c|c|c|c|c|c|c|c|c|}
\hline & & 1998 & 1999 & 2000 & 2001 & 2002 & 2003 & 2004 & 2005 & 2006 & 2007 & 2008 \\
\hline \multicolumn{13}{|l|}{ Males } \\
\hline \multicolumn{13}{|l|}{ Indictable offences } \\
\hline All ages & $\mathbf{K} \mathbf{G A}$ & 142.9 & 126.1 & 109.7 & 103.8 & 104.4 & 109.8 & 110.0 & 129.9 & 147.6 & 148.5 & 132.8 \\
\hline 10 and under 15 years ${ }^{4}$ & $\mathrm{~K}$ GB & 23.7 & 22.0 & 20.3 & 19.7 & 16.7 & 16.9 & 18.7 & 21.0 & 21.7 & 19.7 & 14 \\
\hline 15 and under 18 years ${ }^{4}$ & $\mathrm{~K}$ GC & 32.0 & 28.7 & 25.0 & 24.5 & 23.3 & 24.1 & 25.9 & 28.0 & 30.2 & 30.0 & 24 \\
\hline 18 and under 21 years & K $\mathbf{G}$ & 25.7 & 22.7 & 20.1 & 18.5 & 18.9 & 19.4 & 16.7 & 19.8 & 22.9 & 23.7 & 21.6 \\
\hline 21 years and over & K G & 61.5 & 52.7 & 44.3 & 41.2 & 45.6 & 49.4 & 48.7 & 61.1 & 72.8 & 75.1 & 72 \\
\hline All ages & K $\mathbf{G}$ & 76.9 & 76.1 & 69.6 & 68.0 & 63.8 & 70.9 & 76.0 & 87.6 & 112.0 & 120.2 & 112 \\
\hline 10 and under 15 years ${ }^{4}$ & K GG & 10.6 & 11.7 & 12.0 & 12.7 & 10.3 & 10.9 & 12.6 & 15.5 & 17.9 & 17.9 & 12 \\
\hline 15 and under 18 years ${ }^{4}$ & $\mathrm{~K}$ GH & 16.1 & 16.1 & 14.9 & 15.2 & 13.3 & 15.1 & 17.2 & 18.6 & 21.3 & 21.3 & 16 \\
\hline 18 and under 21 years & K GI & 13.2 & 13.0 & 11.9 & 11.0 & 11.0 & 12.4 & 12.3 & 12.2 & 15.0 & 16.4 & 16 \\
\hline 21 years and over & K $\mathbf{G}$ & 37.0 & 35.3 & 30.9 & 29.0 & 29.2 & 32.5 & 33.9 & 41.2 & 57.8 & 64.6 & 66 \\
\hline
\end{tabular}

\begin{tabular}{|c|c|c|c|c|c|c|c|c|c|c|c|c|}
\hline \multicolumn{13}{|l|}{$\begin{array}{l}\text { Females } \\
\text { Indictable offences }\end{array}$} \\
\hline All ages & $\mathbf{K}$ GK & 48.8 & 44.5 & 41.2 & 40.1 & 38.5 & 41.0 & 46.3 & 53.0 & 56.2 & 56.6 & 47.6 \\
\hline 10 and under 15 years ${ }^{4}$ & $\mathbf{K} \mathbf{G}$ & 11.1 & 9.8 & 10.0 & 10.1 & 8.4 & 8.6 & 10.6 & 12.2 & 12.5 & 12.5 & 9.0 \\
\hline 15 and under 18 years ${ }^{4}$ & K GM & 10.3 & 9.3 & 9.0 & 9.3 & 8.3 & 9.1 & 10.7 & 12.2 & 12.7 & 13.0 & 10.1 \\
\hline 21 years and over & K GO & 21.4 & 19.6 & 17.0 & 15.9 & 17.0 & 18.4 & 19.9 & 22.8 & 24.7 & 24.8 & 23.0 \\
\hline \multicolumn{13}{|l|}{ Summary offences } \\
\hline All ages & K GP & 19.2 & 19.4 & 18.5 & 18.0 & 18.6 & 20.2 & 23.5 & 28.5 & 34.2 & 37.6 & 34.3 \\
\hline 10 and under 15 years ${ }^{4}$ & $\mathrm{~K}$ GQ & 2.1 & 2.5 & 2.8 & 2.9 & 2.7 & 3.0 & 3.9 & 5.1 & 5.6 & 5.6 & 4.4 \\
\hline 15 and under 18 years ${ }^{4}$ & K GR & 3.7 & 3.9 & 3.7 & 3.8 & 3.6 & 4.3 & 5.4 & 6.2 & 7.1 & 7.3 & 6.2 \\
\hline 18 and under 21 years & K GS & 2.6 & 2.7 & 2.5 & 2.3 & 2.4 & 2.7 & 2.9 & 3.3 & 4.1 & 4.7 & 4.5 \\
\hline 21 years and over & K GT & 10.8 & 10.3 & 9.6 & 9.0 & 9.8 & 10.2 & 11.3 & 13.9 & 17.4 & 20.0 & 19.1 \\
\hline
\end{tabular}

1 See chapter text.

2 These data are on the prinicpal offence basis.

3 Every effort is made to ensure that the figures presented are accurate and

As a consequence, care should be taken to ensure data collection processes and their inevitable limitations are taken into account when those data are used.

complete. However, it is important to note that these data have been ex- 4 From 1 June 2000 the Crime and Disorder Act 1998 came into force nationally tracted from large administrative data systems generated by police forces.

and removed the use of cautions for persons under 18 and replaced them with reprimands and warnings. These are included in the totals. 
Crime and justice

Percentages and thousands

\begin{tabular}{|c|c|c|c|c|c|c|c|c|c|c|c|c|}
\hline & & 1998 & 1999 & 2000 & 2001 & 2002 & 2003 & 2004 & 2005 & 2006 & 2007 & 2008 \\
\hline \multicolumn{13}{|l|}{ Males } \\
\hline \multicolumn{13}{|l|}{ Sentence or order } \\
\hline Absolute discharge & KEJB & 0.7 & 0.6 & 0.6 & 0.6 & 0.8 & 0.9 & 0.8 & 0.7 & 0.7 & 0.7 & 0.6 \\
\hline Conditional discharge & KEJC & 15.3 & 15.0 & 14.1 & 13.4 & 12.4 & 13.0 & 12.2 & 11.9 & 11.3 & 12.2 & 11.4 \\
\hline Fine & KEJF & 28.4 & 27.7 & 25.7 & 24.5 & 23.9 & 24.0 & 20.9 & 19.4 & 17.4 & 16.2 & 16.0 \\
\hline Community rehabilitation order & KEJD & 10.0 & 10.1 & 10.1 & 10.7 & 10.6 & 10.1 & 9.5 & 5.6 & 0.8 & 0.5 & 0.4 \\
\hline Supervision order & KEJE & 2.7 & 2.7 & 2.4 & 2.3 & 2.1 & 1.8 & 2.0 & 2.1 & 2.3 & 2.4 & 1.9 \\
\hline Community punishment order & KEJG & 9.3 & 9.3 & 9.5 & 9.0 & 8.6 & 8.3 & 8.8 & 6.3 & 1.2 & 0.5 & 0.4 \\
\hline $\begin{array}{l}\text { Community punishment and } \\
\text { rehabilitation order }\end{array}$ & $\mathrm{KIJW}$ & 3.8 & 3.7 & 3.6 & 2.6 & 2.6 & 2.6 & 2.8 & 2.1 & 0.5 & 0.4 & 0.4 \\
\hline Curfew order & LUJP & 0.2 & 0.3 & 0.5 & 0.7 & 1.1 & 1.6 & 2.7 & 2.3 & 1.3 & 1.3 & 1.4 \\
\hline Reparation order & SNFI & .. & .. & 0.7 & 1.3 & 0.8 & 0.4 & 0.4 & 0.5 & 0.6 & 0.6 & 0.6 \\
\hline Action plan order & SNFJ & .. & .. & 0.9 & 1.7 & 1.1 & 0.7 & 0.8 & 0.8 & 0.8 & 0.8 & 0.7 \\
\hline Drug treatment and testing order & SNFK & .. & .. & 0.1 & 1.2 & 1.4 & 1.9 & 2.3 & 1.6 & 0.1 & - & 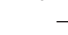 \\
\hline Referral order & SNFL & .. & .. & .. & .. & 3.0 & 4.0 & 4.4 & 5.2 & 5.2 & 5.5 & 5.0 \\
\hline Community order ${ }^{2}$ & GN7P & & 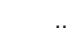 & .. &.. & & & & 8.7 & 19.8 & 20.4 & 20.7 \\
\hline $\begin{array}{l}\text { Suspended sentence order } \\
\text { Imprisonment }\end{array}$ & KEJL & 0.7 & 0.6 & 0.7 & 0.6 & 0.5 & 0.5 & 0.0 & 1.7 & 6.7 & 8.6 & 8.9 \\
\hline Detention and training order & LUJR & - & 0.1 & 1.4 & 1.9 & 1.8 & 1.5 & 1.6 & 1.6 & 1.7 & 1.5 & 1.5 \\
\hline Young offender institution & KEJK & 6.0 & 6.2 & 5.2 & 4.5 & 4.2 & 3.6 & 3.8 & 3.7 & 3.6 & 3.8 & 3.6 \\
\hline Unsuspended imprisonment & KEJM & 18.2 & 18.7 & 19.9 & 20.0 & 20.9 & 20.6 & 21.5 & 21.2 & 20.4 & 19.9 & 21.6 \\
\hline Other sentence or order & KEJN & 2.6 & 3.1 & 3.1 & 3.4 & 3.3 & 3.5 & 4.1 & 3.9 & 4.4 & 4.0 & 4.4 \\
\hline \multicolumn{13}{|l|}{ Total number of males } \\
\hline (thousands) $=100$ per cent & KEJA & 292.4 & 291.3 & 277.1 & 274.6 & 285.6 & 282.3 & 267.5 & 259.4 & 258.4 & 265.8 & 266.9 \\
\hline \multicolumn{13}{|l|}{ Females } \\
\hline \multicolumn{13}{|l|}{ Sentence or order } \\
\hline Absolute discharge & KEKB & 0.7 & 0.7 & 0.6 & 0.6 & 0.9 & 1.0 & 0.8 & 0.8 & 0.7 & 0.8 & 0.7 \\
\hline Conditional discharge & KEKC & 28.7 & 26.9 & 24.9 & 23.9 & 22.0 & 22.5 & 21.8 & 20.7 & 20.1 & 20.8 & 20.0 \\
\hline Fine & KEKF & 21.3 & 20.8 & 20.1 & 18.6 & 17.9 & 18.5 & 16.7 & 15.2 & 12.8 & 12.1 & 11.9 \\
\hline Community rehabilitation order & KEKD & 19.1 & 19.4 & 19.6 & 19.1 & 19.2 & 17.0 & 15.4 & 9.0 & 1.4 & 0.5 & 0.4 \\
\hline Supervision order & KEKE & 3.1 & 2.9 & 2.8 & 2.7 & 2.1 & 2.1 & 2.1 & 2.4 & 2.5 & 2.6 & 2.0 \\
\hline Community punishment order & KEKG & 6.5 & 7.1 & 7.5 & 7.3 & 6.8 & 6.6 & 7.6 & 6.1 & 1.6 & 0.4 & 0.2 \\
\hline Attendance centre order & KEKH & 0.9 & 0.9 & 0.8 & 0.6 & 0.4 & 0.3 & 0.3 & 0.3 & 0.5 & 0.3 & 0.3 \\
\hline Reparation order & SNFX & .. & .. & 0.8 & 1.6 & 0.8 & 0.4 & 0.5 & 0.5 & 0.5 & 0.6 & 0.6 \\
\hline Action plan order & SNFZ & .. & .. & 1.0 & 2.0 & 1.2 & 0.8 & 0.8 & 1.0 & 0.9 & 0.9 & 0.7 \\
\hline Drug treatment and testing order & SNGA & .. & .. & 0.1 & 1.4 & 1.7 & 2.4 & 3.2 & 2.1 & 0.2 & - & 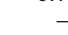 \\
\hline Referral order & SNGB & .. & .. & .. & .. & 3.9 & 5.1 & 5.6 & 6.7 & 6.9 & 7.0 & 6.2 \\
\hline Community order ${ }^{2}$ & GN7Q & .. & .. & .. &.. & .. & .. & .. & 9.9 & 23.3 & 24.5 & 25.4 \\
\hline $\begin{array}{l}\text { Suspended sentence order } \\
\text { Imprisonment }\end{array}$ & KEKL & 1.5 & 1.3 & 1.3 & 1.2 & 1.1 & 1.0 & 1.3 & 2.5 & 7.8 & 9.6 & 10.1 \\
\hline Sec $90-92$ & LUJU & .. & 0.1 & 0.1 & 0.1 & 0.1 & 0.1 & 0.1 & 0.1 & - & - & - \\
\hline Detention and training order & LUJV & - & - & 0.6 & 0.8 & 0.8 & 0.7 & 0.7 & 0.8 & 0.7 & 0.8 & 0.7 \\
\hline Young offender institution & KEKK & 2.2 & 2.4 & 2.2 & 2.0 & 1.9 & 1.7 & 1.4 & 1.6 & 1.6 & 1.4 & 1.3 \\
\hline Unsuspended imprisonment & KEKM & 10.0 & 11.0 & 11.5 & 12.1 & 12.7 & 12.8 & 13.2 & 12.6 & 12.8 & 12.4 & 13.5 \\
\hline Other sentence or order & KEKN & 2.5 & 3.0 & 2.9 & 3.5 & 3.4 & 3.8 & 4.3 & 3.8 & 4.3 & 3.7 & 4.7 \\
\hline $\begin{array}{l}\text { Total number of females } \\
\text { (thousands) }=100 \text { per cent }\end{array}$ & KEKA & 47.2 & 49.0 & 47.8 & 47.3 & 49.9 & 50.2 & 48.3 & 46.1 & 43.7 & 45.4 & 46.3 \\
\hline
\end{tabular}

1 See chapter text. Every effort is made to ensure that the figures presented be taken to ensure data collection processes and their inevitable limitations are are accurate and complete. However, it is important to note that these data taken into account when those data are used.

have been extracted from large administrative data systems generated by 2 The community order was introduced on 4 April 2005 and applies to offences police forces. As a consequence, care should committed on or after that date. 


\section{Persons sentenced to life imprisonment or immediate custody: by sex and age \\ England and Wales}

Number of Persons

\begin{tabular}{|c|c|c|c|c|c|c|c|c|c|c|c|c|}
\hline & & 1998 & 1999 & 2000 & 2001 & 2002 & 2003 & 2004 & 2005 & 2006 & 2007 & 2008 \\
\hline \multirow{2}{*}{\multicolumn{13}{|c|}{ Life imprisonment ${ }^{1}$}} \\
\hline \multicolumn{12}{|l|}{ Males } & \\
\hline All ages & I28G & 380 & 465 & 446 & 484 & 536 & 489 & 548 & 594 & 531 & 471 & 495 \\
\hline $10-17$ years & I28D & 11 & 26 & 19 & 28 & 21 & 11 & 15 & 27 & 16 & 23 & 24 \\
\hline $18-20$ years & I28E & 25 & 38 & 9 & 27 & 21 & 47 & 24 & 50 & 46 & 70 & 54 \\
\hline 21 years and over & I28F & 344 & 401 & 418 & 429 & 494 & 431 & 509 & 517 & 469 & 378 & 417 \\
\hline \multicolumn{13}{|l|}{ Females } \\
\hline All ages & I28K & 14 & 19 & 21 & 19 & 19 & 24 & 22 & 31 & 16 & 21 & 28 \\
\hline $10-17$ years & I $28 \mathrm{H}$ & 1 & 3 & 2 & 1 & 1 & - & 1 & 1 & - & 3 & 1 \\
\hline $18-20$ years & I28I & - & 2 & 1 & 3 & 2 & 4 & 2 & 4 & 2 & 3 & 2 \\
\hline 21 years and over & $I 28 \mathrm{~J}$ & 13 & 14 & 18 & 15 & 16 & 20 & 19 & 26 & 14 & 15 & 25 \\
\hline \multicolumn{13}{|l|}{ All persons } \\
\hline All ages & I280 & 394 & 484 & 467 & 503 & 555 & 513 & 570 & 625 & 547 & 492 & 523 \\
\hline $10-17$ years & I28I & 12 & 29 & 21 & 29 & 22 & 11 & 16 & 28 & 16 & 26 & 25 \\
\hline 18 - 20 years & I28M & 25 & 40 & 10 & 30 & 23 & 51 & 26 & 54 & 48 & 73 & 56 \\
\hline 21 years and over & I2 $8 \mathrm{~N}$ & 357 & 415 & 436 & 444 & 510 & 451 & 528 & 543 & 483 & 393 & 442 \\
\hline \multicolumn{13}{|l|}{ Immediate custody ${ }^{2}$} \\
\hline \multicolumn{13}{|l|}{ Males } \\
\hline All ages & JF7E & 93619 & 97355 & 97841 & 97728 & 102240 & 98371 & 97020 & 91954 & 86239 & 85285 & 88826 \\
\hline $10-17$ years & JF7F & 6870 & 7218 & 6949 & 7119 & 6865 & 5765 & 5866 & 5463 & 5669 & 5277 & 4939 \\
\hline $18-20$ years & JF7G & 16127 & 17011 & 17315 & 16855 & 16269 & 14418 & 13793 & 13237 & 12802 & 13126 & 12354 \\
\hline 21 years and over & JF7H & 70622 & 73126 & 73577 & 73754 & 79106 & 78188 & 77361 & 73254 & 67768 & 66882 & 71533 \\
\hline \multicolumn{13}{|l|}{ Females } \\
\hline All ages & JF7I & 6553 & 7485 & 7879 & 8042 & 8812 & 8786 & 8732 & 8231 & 7783 & 7722 & 8284 \\
\hline $10-17$ years & JF7J & 335 & 406 & 444 & 448 & 529 & 424 & 443 & 498 & 453 & 466 & 442 \\
\hline 18 - 20 years & JF7K & 851 & 961 & 1116 & 1063 & 1071 & 969 & 817 & 875 & 841 & 793 & 782 \\
\hline 21 years and over & JF7L & 5367 & 6118 & 6319 & 6531 & 7212 & 7393 & 7472 & 6858 & 6489 & 6463 & 7060 \\
\hline \multicolumn{13}{|l|}{ All persons } \\
\hline All ages & JF7M & 100172 & 104840 & 105720 & 105770 & 111052 & 107157 & 105752 & 100185 & 94022 & 93007 & 97464 \\
\hline $10-17$ years & JF7N & 7205 & 7624 & 7393 & 7567 & 7394 & 6189 & 6309 & 5961 & 6122 & 5743 & 5403 \\
\hline $18-20$ years & JF70 & 16978 & 17972 & 18431 & 17918 & 17340 & 15387 & 14610 & 14112 & 13643 & 13919 & 13180 \\
\hline 21 years and over & JF7P & 75989 & 79244 & 79896 & 80285 & 86318 & 85581 & 84833 & 80112 & 74257 & 73345 & 78881 \\
\hline
\end{tabular}

1 Includes detention under the Powers of Criminal Courts (Sentencing) Act Sec 109 (persons aged 18 and over) and immediate imprisonment (persons 2000 , Secs 90-92 (Childrens and Young Persons Act 1993, Secs 53(1) \&

(2) prior to Aug 2000) (persons aged 10-17), custody for life under the aged 21 and over). Indeterminate sentences for public protection under the

sons aged 18 - 20), mandatory life sentences under the Powers of Criminal

Courts (Sentencing) Act 2000

Criminal Justice Act 2003 are excluded.

2 Excludes life and indeterminate sentences.

Source: Justice Statistics Analytical Services in the Ministry of Justice 020 
Numbers ${ }^{1}$

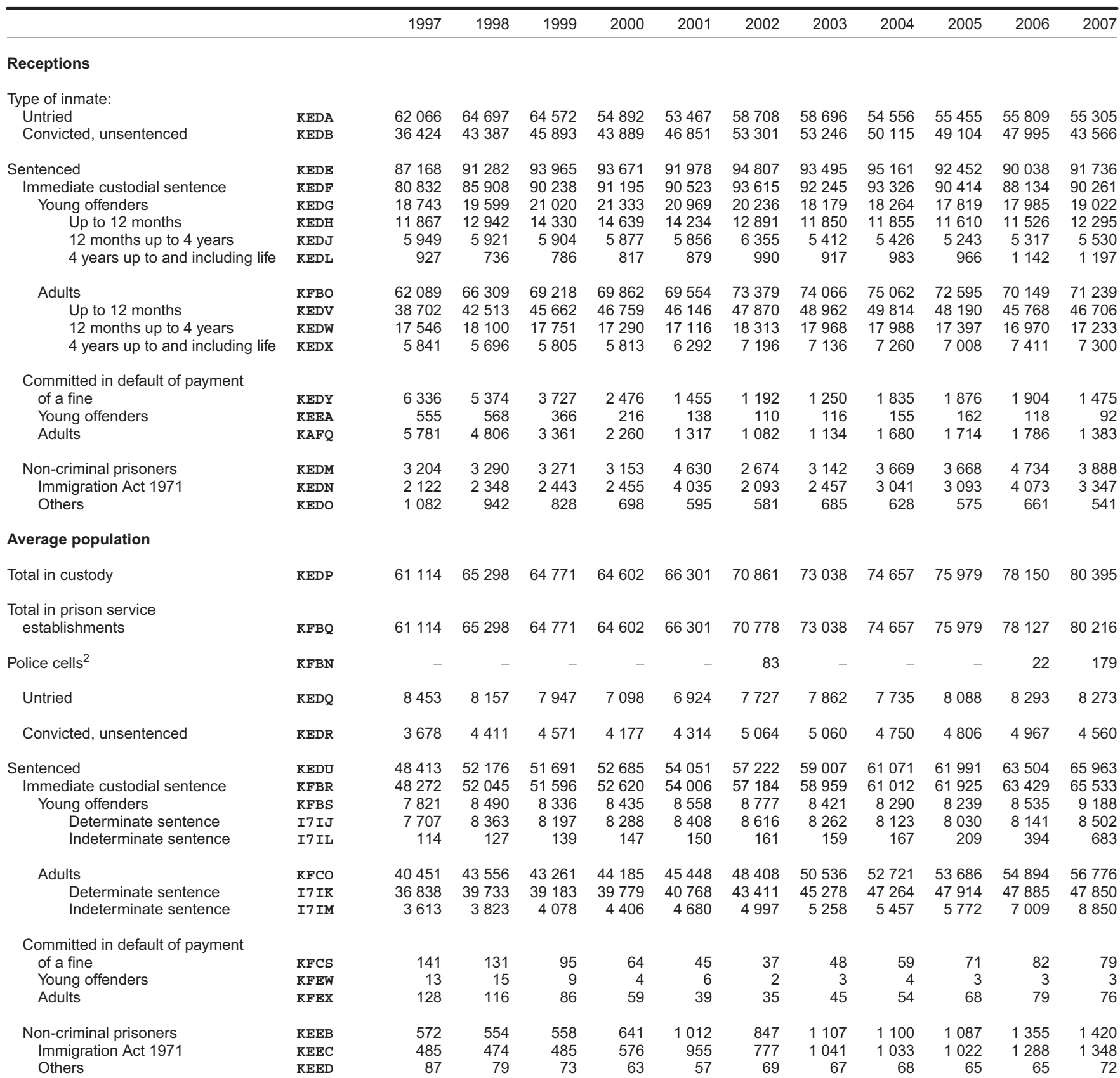

3 In use Certified Normal Accommodation at 30 June every year. 


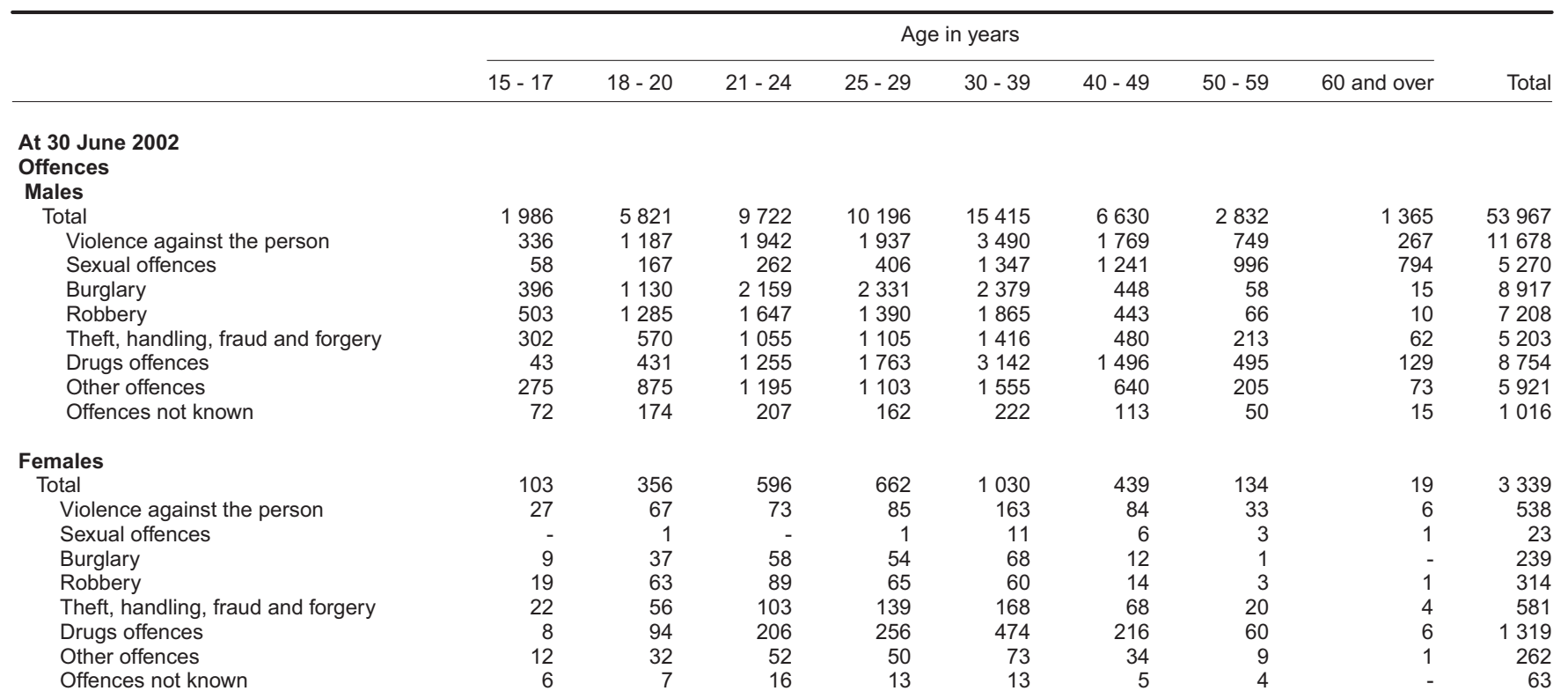

At 30 June 2003

Offences

Males

Total

Violence against the person

Sexual offences

Burglary

Robbery

Theft, handling, fraud and forgery
Drugs offences

Other offences

Offences not known

1724

310

5740

1257

183

1370

1370
543

543
452

884

133

2112

310

2003
1910

1910
1020

1256

1329

172

10441

2068

390

2204

1546
1060

1060
1791

1218

164

16304

3733

1376

2555

2022

1437

3215

1760

205

7252

1932

1353

527

514

472
1579

787

89

$1100 \quad 492 \quad 123$

155
11
77
93
199
453
108
5

Violence against the person

Sexual offences

Burglary

Robbery

Theft, handling, fraud and forgery

Drugs offences

Other offences

Offences not known

$\begin{array}{rr}670 & 702 \\ 91 & \\ 2 & \\ 64 & \\ 105 & 1 \\ 117 & 128 \\ 226 & 271 \\ 58 & \\ 8 & \end{array}$

2975
780
1023
71
69
201
528
263

$\begin{array}{rr}1413 & 55962 \\ 290 & 12482 \\ 838 & 5514 \\ 11 & 8579 \\ 12 & 7879 \\ 45 & 5069 \\ 127 & 8993 \\ 69 & 6581 \\ 21 & 865\end{array}$

3477

506

26

240
407

609

1343

1343
311

At 30 June 2004

Offences

Males

Total

Violence against the person

Sexual offences

Burglary

Robbery

Theft, handling, fraud and forgery

Drugs offences

Other offences

Offences not known

1706
326
55
242
449
272
51
286
25

5585
1353
193
855
1254
502
471
848
108

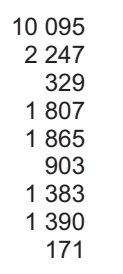

10738
2272
424
2141
1691
1045
1789
1249
126

17021
3965
1433
2662
2127
1479
3258
1895
202

7858
2107
1416
608
583
573
1615
868
87

3013
799
1030
71
70
180
537
284
41

Females

Violence against the person

Sexual offences

Burglary

Robbery

Theft, handling, fraud and forgery

Drugs offences

Other offences

Offences not known

$\begin{array}{rrrrrrrrr}58 & 300 & 632 & 727 & 1056 & 507 & 152 & 20 & 3453 \\ 15 & 70 & 98 & 89 & 192 & 95 & 36 & 9 & 603 \\ - & - & 3 & 3 & 8 & 7 & 4 & 2 & 27 \\ 6 & 19 & 59 & 83 & 56 & 22 & 3 & - & 247 \\ 8 & 65 & 93 & 90 & 114 & 20 & 2 & - & 392 \\ 11 & 28 & 100 & 140 & 171 & 67 & 25 & 1 & 543 \\ 6 & 78 & 197 & 245 & 392 & 246 & 65 & 6 & 1235 \\ 11 & 37 & 75 & 72 & 108 & 44 & 13 & 2 & 361 \\ 2 & 3 & 8 & 7 & 15 & 7 & 4 & - & 46\end{array}$


Age in years

$\begin{array}{llllllll}15-17 & 18-20 & 21-24 & 25-29 & 30-39 & 40-49 & 50-59 & 60 \text { and over } \quad \text { Total }\end{array}$

At 30 June 2005

Offences

Males

Total

Violence against the person

Sexual offences

Burglary

Robbery

Theft, handling, fraud and forgery

Drugs offences

Other offences

Offences not known

Females

Total

Violence against the person

Sexual offences

Burglary

Robbery

Theft, handling, fraud and forgery

Drugs offences

Other offences

Offences not known

1782
366
65
285
422
240
76
310
18

5595
1493
186
719
1307
433
491
870
96

$\begin{array}{rr}9937 & 10969 \\ 2553 & 2553 \\ 397 & 505 \\ 1559 & 1947 \\ 1819 & 1705 \\ 858 & 1074 \\ 1332 & 1834 \\ 1306 & 1245 \\ 113 & 106\end{array}$

16843
4015
1436
2570
2035
1449
3263
1902
173

8731

2402

1552
669

669

649

650

1741

987
81

3256
840
1084
78
83
238
544
360
29

$1594 \quad 58707$

$319 \quad 14541$

$\begin{array}{rr}922 & 6147 \\ 17 & 7844\end{array}$

$15 \quad 8035$

$55 \quad 4997$

$148 \quad 9429$

997079

7079
635

$\begin{array}{rrr}269 & 614 & 680 \\ 68 & 109 & \\ 2 & 3 & \\ 18 & 50 & \\ 59 & 61 & \\ 35 & 105 & 1 \\ 54 & 195 & 25 \\ 30 & 84 & \\ 3 & 7 & \end{array}$

$\begin{array}{rr}680 & 1073 \\ 85 & 190 \\ 4 & 12 \\ 62 & 79 \\ 82 & 102 \\ 119 & 202 \\ 255 & 366 \\ 68 & 117 \\ 5 & \end{array}$

1073
190
12
79
102
202
366
117
5

\section{5}

$114 \quad 40$

8

23
20

88

268

56

1814
381
67
275
486
200
68
327
12

5716

1563

213
707

1413

1413
451

451
492
835

835

2616
452

1363

1739

830
1232

1326

Other offences

Offences not known

55

11349

2977
560

1838

1674

1093

1913
1231

631
64

16828

4109

1497

2554

1975

1598

3153

105

9349

2609

1683

715
706

706
707

1829

1040

60

3511
935
1118
97
91
214
623
406
28

1719

$\begin{array}{rr}348 & 15537 \\ 971 & 6561\end{array}$

$15 \quad 7563$

$\begin{array}{ll}8 & 100 \\ 5 & 147\end{array}$

9484

7129

378

Females

Total

Violence against the person

Sexual offences

Burglary

Robbery

Theft, handling, fraud and forgery

Drugs offences

Other offences

Offences not known

$\begin{array}{rrrr}50 & 271 & 551 & 707 \\ 11 & 75 & 111 & 101 \\ 1 & 3 & 2 & \\ 7 & 13 & 39 & \\ 17 & 48 & 67 & \\ 3 & 30 & 97 & 171 \\ 4 & 62 & 158 & 2 \\ 7 & 36 & 61 & \\ 1 & 5 & 5 & \end{array}$

$\begin{array}{rr}707 & 1094 \\ 101 & 205 \\ 2 & \\ 63 & \\ 76 & \\ 171 & 236 \\ 217 & 354 \\ 72 & 120 \\ 4 & \end{array}$

$\begin{array}{rrr}1094 & 604 & 18 \\ 205 & 118 & 48 \\ 8 & 13 & \\ 80 & 24 & \\ 86 & 18 & \\ 232 & 106 & 2 \\ 354 & 253 & 8 \\ 120 & 67 & 19 \\ 9 & 5 & \end{array}$

3506

678

37
228

315

671

1163

385

30

At 30 June 2007

Offences

Males
Total

Violence against the person

Sexual offences

Burglary

Robbery

Theft, handling, fraud and forgery

Drugs offences

Other offences

Offences not known

1827
402
73
281
495
194
71
294
17

6354
1772
276
817
1558
426
546
908
51

9860
2927
487
1334
1855
677
1285
1237
59

11653
3183
675
1865
1716
1044
1858
1242
69

16606
4337
1555
2492
1928
1478
3002
1731
83

$\begin{array}{rr}10092 & 3823 \\ 2846 & 1063 \\ 1909 & 1202 \\ 812 & 109 \\ 774 & 96 \\ 711 & 246 \\ 1908 & 689 \\ 1087 & 401 \\ 47 & 18\end{array}$

$\begin{array}{rr}1973 & 62188 \\ 399 & 16929 \\ 1112 & 7287 \\ 13 & 7723 \\ 14 & 8437 \\ 67 & 4844 \\ 210 & 9569 \\ 152 & 7051 \\ 5 & 348\end{array}$

Females

Violence against the person

Sexual offences

Burglary

Robbery

Theft, handling, fraud and forgery

Drugs offences

Other offences

Offences not known

$\begin{array}{rr}280 & 475 \\ 99 & 113 \\ 1 & 3 \\ 11 & 28 \\ 54 & 53 \\ 29 & 68 \\ 42 & 124 \\ 45 & 75 \\ - & 10\end{array}$

\begin{tabular}{rrrrrr}
662 & 1011 & 610 & 202 & 49 & 3345 \\
111 & 170 & 117 & 47 & 11 & 687 \\
7 & 16 & 15 & 4 & 2 & 48 \\
56 & 76 & 23 & 1 & - & 197 \\
78 & 82 & 27 & 2 & 1 & 311 \\
128 & 211 & 111 & 37 & 10 & 601 \\
201 & 335 & 230 & 90 & 19 & 1044 \\
76 & 115 & 77 & 20 & 6 & 423 \\
5 & 6 & 10 & 2 & - & 35 \\
\hline
\end{tabular}

1 The data presented in this table are drawn from administrative IT systems.

Where figures in the table have been rounded to the nearest whole number,

the rounded components do not always add to the totals, which are calcu-

lated and rounded independently. Reconciliation exercises with published

Home Office figures may demonstrate differences due to rounded compo-

nents. A programme of work is currently being undertaken to audit the

quality of the data and to identify priorities for improvements.

2 Excludes persons committed in default of payment of a fine. 
Operating cost and total capital employed, years ending 31 March

\begin{tabular}{|c|c|c|c|c|c|c|c|c|}
\hline & & $\begin{array}{r}2001 \\
/ 02\end{array}$ & $\begin{array}{r}2002 \\
/ 03\end{array}$ & $\begin{array}{r}2003 \\
/ 04\end{array}$ & $\begin{array}{r}2004 \\
/ 05\end{array}$ & $\begin{array}{r}2005 \\
/ 06\end{array}$ & $\begin{array}{r}2006 \\
/ 07\end{array}$ & $\begin{array}{r}2007 \\
108\end{array}$ \\
\hline \multicolumn{9}{|l|}{ Expenditure } \\
\hline Staff costs & KWUV & 1138400 & 1259502 & 1364193 & 1439882 & 1498446 & 1586126 & 1646757 \\
\hline Accommodation costs & $\mathrm{KXCO}$ & 193100 & 200000 & 194000 & 185400 & 150270 & 138322 & 144170 \\
\hline Other operating costs & $\mathrm{KXCP}$ & 706100 & 756198 & 653007 & 694618 & 528529 & 498913 & 511688 \\
\hline Depreciation & $\mathrm{KXCQ}$ & 128100 & 132600 & 129600 & 143800 & 7974 & 11044 & 12135 \\
\hline Cost of capital & $\mathrm{KXCR}$ & 284900 & 292700 & 164400 & 170300 & 279 & 905 & -610 \\
\hline Total expenditure & $\mathrm{KXCS}$ & 2450600 & 2641000 & 2505200 & 2634000 & 2185498 & 2235310 & 2314140 \\
\hline \multicolumn{9}{|l|}{ Income } \\
\hline Contributions from industries & $\mathrm{KXCT}$ & -11600 & -10100 & -11000 & -10600 & -11154 & -7698 & -7079 \\
\hline Other operating income & $\mathrm{KXCU}$ & -13100 & -15500 & -21000 & -38400 & -41323 & -45411 & -43566 \\
\hline Income from Other Government Departments ${ }^{1}$ & GDPM & -180600 & -210200 & -368000 & -381500 & -302549 & -245917 & -205524 \\
\hline Total income & $\mathrm{KXCV}$ & -205300 & -235800 & -400000 & -430500 & -355026 & -299026 & -256169 \\
\hline Net operating costs & $\mathrm{KXCW}$ & 2245300 & 2405200 & 2105200 & 2203500 & 1830472 & 1936284 & 2057971 \\
\hline Total capital employed & $\mathrm{KXCX}$ & 4859600 & 4821500 & 5228600 & 5116700 & 5716 & -52207 & -42577 \\
\hline
\end{tabular}

1 Income from the Youth Justice Board (a non-departmental public body of Source: NOMS Agency: 02072175213

the Home Office) for the provision of juvenile custody within the Prison Ser-

vice, Department for Education and Skills for the provision of education ser-

vices and Department of Health and PCTs for the provision of healthcare. 


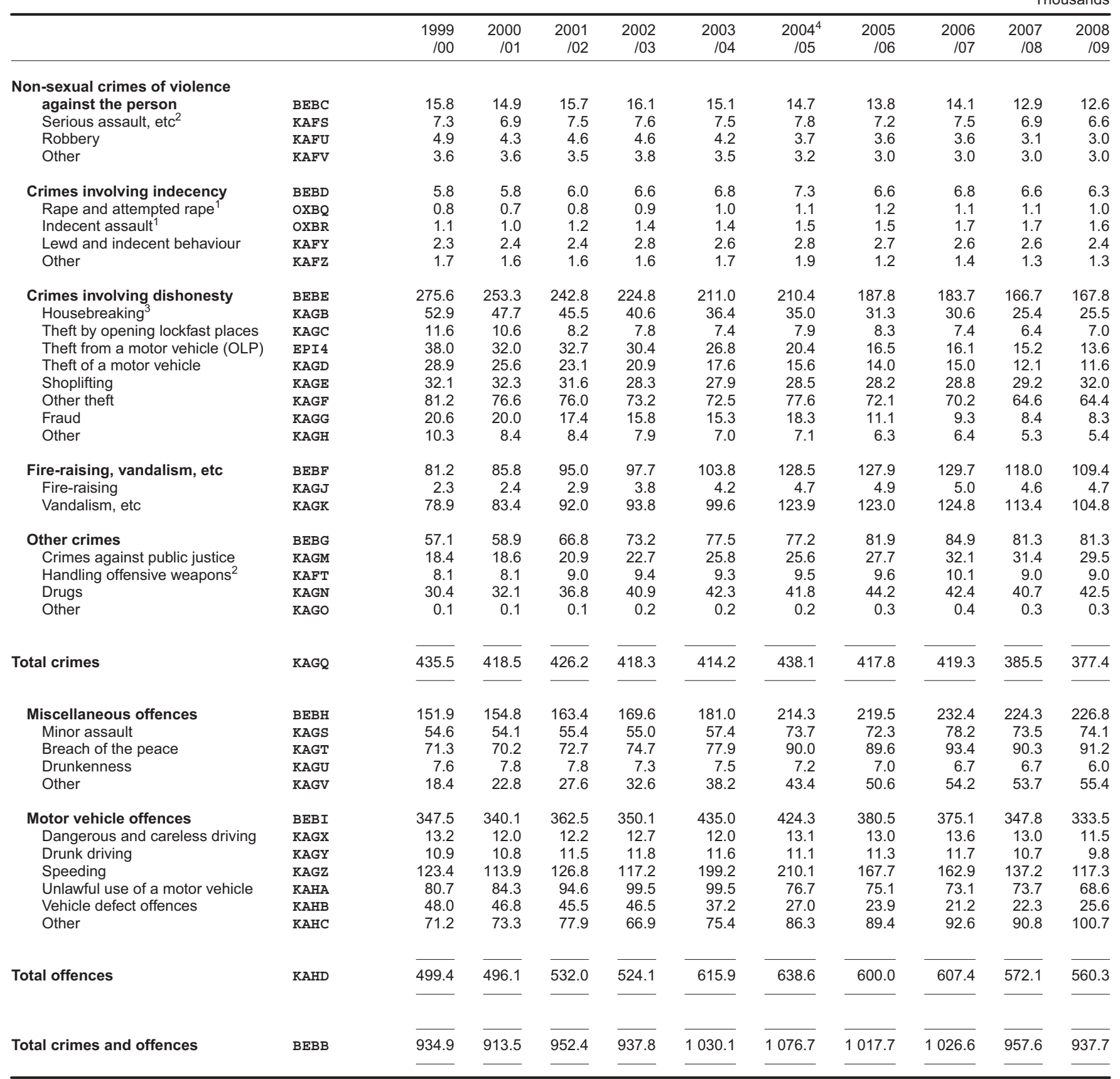

1 See chapter text.

Source: The Scottish Government Justice Department: 01312442635

2 Includes murder, attempted murder, culpable homicide and serious assault.

3 Includes dwellings, non-dwellings and other premises.

4 The introduction of the Scottish Crime Recording Standard on 1 April 2004

has increased the number of minor crimes recorded, such as minor crimes

of theft, vandalism, petty assault and breach of the peace. 
Numbers

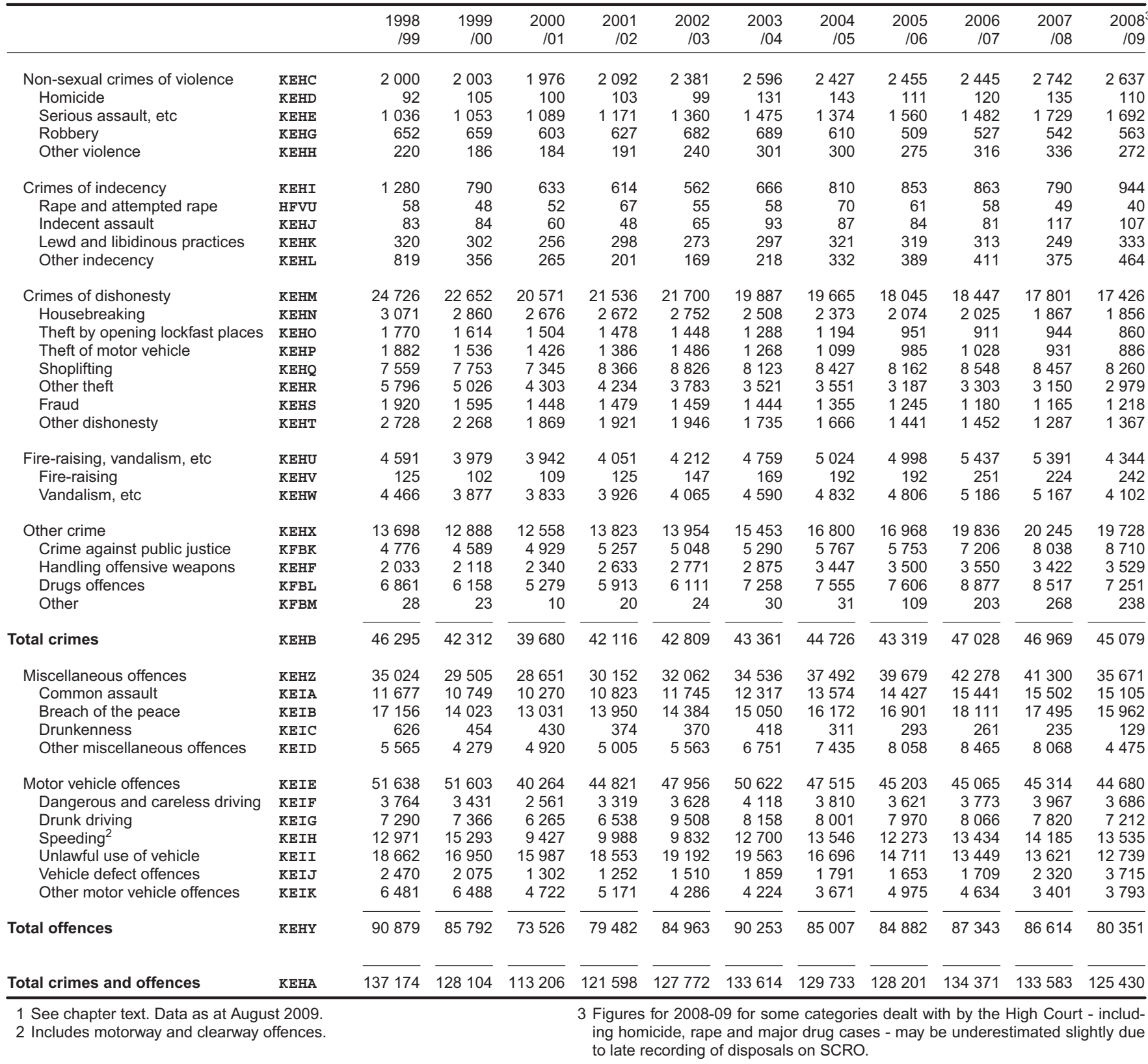

Source: Scottish Government Justice Department: 01312442229

\section{Persons with a charge proved: by court procedure ${ }^{1,2}$ Scotland}

\begin{tabular}{rrrrrrrrrrrr}
\hline Numbers \\
\hline & 1998 & 1999 & 2000 & 2001 & 2002 & 2003 & 2004 & 2005 & 2006 & 2007 & 2008 \\
109 & 100 & 101 & 102 & 103 & 104 & 105 & 106 & $/ 07$ & 108 & 109 \\
\hline
\end{tabular}

\section{Court procedure}

\begin{tabular}{|c|c|c|c|c|c|c|c|c|c|c|c|c|}
\hline High Court ${ }^{3}$ & KEIQ & 1043 & 1174 & 1092 & 1125 & 1194 & 1217 & 974 & 882 & 866 & 837 & 784 \\
\hline Sheriff Court & KEIU & 74484 & 70541 & 65714 & 72021 & 80117 & 80155 & 80866 & 79956 & 85185 & 85175 & 78200 \\
\hline District Court ${ }^{4,5}$ & KEIV & 50784 & 46052 & 38422 & 38484 & 41516 & 47144 & 47891 & 47358 & 48319 & 47569 & 46430 \\
\hline Stipendiary Magistrate Court ${ }^{4}$ & KEIW & 6646 & 5652 & 3365 & 5455 & .. & .. & .. & .. & $\cdot$. & 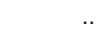 & \\
\hline al called to court ${ }^{6}$ & KEIZ & 132957 & 123420 & 108595 & 117089 & 122827 & 128519 & 129733 & 128201 & 134371 & 133583 & 125430 \\
\hline
\end{tabular}

1 See chapter text.

2 All figures are now reported as financial years.

4 District Court figures from 2002/03 include the Stipendiary Magistrate Court.

3 Including cases remitted to the High Court from the Sheriff Court. Figure for from 10 March 2008.

$2007 / 08$ may be an underestimate due to late recording of disposals on the 6 Includes court type not known.

Scottish Criminal History System. 
Numbers

\begin{tabular}{|c|c|c|c|c|c|c|c|c|c|c|c|c|}
\hline & & $\begin{array}{r}1998 \\
199 \\
\end{array}$ & $\begin{array}{r}1999 \\
100 \\
\end{array}$ & $\begin{array}{r}2000 \\
101 \\
\end{array}$ & $\begin{array}{r}2001 \\
102 \\
\end{array}$ & $\begin{array}{r}2002 \\
103 \\
\end{array}$ & $\begin{array}{r}2003 \\
104 \\
\end{array}$ & $\begin{array}{r}2004 \\
105 \\
\end{array}$ & $\begin{array}{r}2005 \\
106 \\
\end{array}$ & $\begin{array}{r}2006 \\
107 \\
\end{array}$ & $\begin{array}{r}2007 \\
108 \\
\end{array}$ & $\begin{array}{r}2008 \\
109 \\
\end{array}$ \\
\hline \multicolumn{13}{|l|}{ Main penalty } \\
\hline Restriction of liberty order ${ }^{3}$ & ZBRE & 106 & 196 & 152 & 166 & 656 & 879 & 1097 & 1136 & 1179 & 1155 & 1132 \\
\hline Supervised attendance order ${ }^{4}$ & $\mathrm{ZBRF}$ & 31 & 37 & 5 & 11 & 13 & 18 & 33 & 99 & 112 & 129 & 197 \\
\hline Drug treatment and testing order ${ }^{5}$ & OEWA & & 5 & 117 & 286 & 409 & 610 & 713 & 758 & 865 & 822 & 881 \\
\hline Absolute discharge & KEXA & 403 & 368 & 364 & 415 & 385 & 435 & 403 & 401 & 411 & 430 & 412 \\
\hline Admonition or caution & KEXB & 13442 & 12188 & 11203 & 11702 & 12360 & 12934 & 13744 & 14175 & 15967 & 16083 & 16350 \\
\hline Probation & KEXC & 6824 & 6542 & 6654 & 7708 & 8451 & 8137 & 8623 & 8785 & 8613 & 9000 & 9858 \\
\hline Remit to children's hearing & KEXD & 176 & 120 & 116 & 158 & 230 & 196 & 221 & 260 & 313 & 259 & 209 \\
\hline Community service order & KEXE & 4811 & 4254 & 4272 & 4323 & 4719 & 4298 & 4849 & 5195 & 5284 & 5599 & 5779 \\
\hline Fine & KEXF & 91393 & 84255 & 70683 & 76217 & 78541 & 84327 & 83237 & 80723 & 83445 & 82019 & 72571 \\
\hline Compensation order & KEXG & 1238 & 1151 & 1076 & 1142 & 1347 & 1767 & 1695 & 1471 & 1375 & 1325 & 1150 \\
\hline Insanity, hospital, guardianship order & KYAN & 125 & 136 & 116 & 103 & 101 & 129 & 95 & 115 & 65 & 20 & 16 \\
\hline Prison & KEXI & 10635 & 10609 & 10430 & 11437 & 12427 & 11960 & 12307 & 12153 & 13456 & 13575 & 13851 \\
\hline Young offenders' institution & KEXJ & 3749 & 3546 & 3394 & 3407 & 3162 & 2801 & 2685 & 2902 & 3241 & 3141 & 3003 \\
\hline Detention of child & KEXM & 24 & 13 & 13 & 14 & 25 & 24 & 20 & 24 & 24 & 26 & 21 \\
\hline
\end{tabular}

1 See chapter text.

2 All figures are now reported as financial years.

3 A community sentence introduced by Section 5 of the Crime and Punishment (Scotland) Act 1995 and available on a pilot basis to 3 Scottish sheriff courts since August 1998. This sentence was made available to High Court, Sheriff Courts and Stipendiary Magistrates court from 1 May 2002.

4 The pilot scheme under the Crime and Punishment (S) Act 1995, where fines for $16 \& 17$ year olds were replaced by supervised attendance orders, was discontinued in December 1999. The majority of supervised attendance orders recorded from the year 2000-01 onwards were disposals relating to the breach of an existing order.
5 Drug treatment and testing orders are new measures made available on a pilot basis to the High Court and to Sheriff Courts for residents in Glasgow (from October 1999), Fife (from July 2000) and Aberdeen/Aberdeenshire (from December 2001). They are now available to all Sheriff courts and the High Court 6 Totals from 2002/03 include a small number of cases where penalty is unknown.

Source: Scottish Government Justice Department: 01312442229

\section{$147 \begin{aligned} & \text { Persons with charge proved }{ }^{1} \text { : by age and sex } \\ & \text { Scotland }\end{aligned}$}

\begin{tabular}{|c|c|c|c|c|c|c|c|c|c|c|c|c|}
\hline & & $\begin{array}{r}1998 \\
/ 99\end{array}$ & $\begin{array}{r}1999 \\
100\end{array}$ & $\begin{array}{r}2000 \\
/ 01\end{array}$ & $\begin{array}{r}2001 \\
102\end{array}$ & $\begin{array}{r}2002 \\
103\end{array}$ & $\begin{array}{r}2003 \\
104\end{array}$ & $\begin{array}{r}2004 \\
105\end{array}$ & $\begin{array}{r}2005 \\
106\end{array}$ & $\begin{array}{r}2006 \\
107\end{array}$ & $\begin{array}{r}2007 \\
108\end{array}$ & $\begin{array}{r}2008 \\
109\end{array}$ \\
\hline Males & KEWA & 114884 & 106654 & 92919 & 100874 & 104312 & 107932 & 108460 & 107801 & 113472 & 112768 & 105908 \\
\hline Under 16 & KEWB & 112 & 75 & 56 & 80 & 129 & 96 & 107 & 133 & 121 & 156 & 106 \\
\hline 16 to 20 & KEWC & 27399 & 24671 & 21973 & 23701 & 23948 & 23454 & 23098 & 24051 & 25513 & 24366 & 20349 \\
\hline 21 to 30 & KEWD & 43599 & 40048 & 35251 & 38441 & 39405 & 40053 & 39336 & 38078 & 40390 & 41216 & 38764 \\
\hline Over 30 & KEWE & 42857 & 41047 & 34957 & 38362 & 40811 & 44324 & 45913 & 45536 & 47446 & 47030 & 46689 \\
\hline Females & KEWG & 17405 & 16188 & 15302 & 15871 & 18160 & 20120 & 20775 & 20039 & 20599 & 20560 & 19511 \\
\hline Under 16 & KEWH & 2 & 5 & 10 & 4 & 5 & 17 & 18 & 8 & 9 & 3 & 18 \\
\hline 16 to 20 & KEWI & 3252 & 3089 & 2768 & 2742 & 2840 & 2927 & 2891 & 2929 & 3255 & 3302 & 2802 \\
\hline 21 to 30 & KEWJ & 6872 & 6219 & 5833 & 6200 & 6843 & 7494 & 7652 & 7387 & 7398 & 7385 & 7281 \\
\hline Over 30 & KEWK & 6971 & 6614 & 6448 & 6854 & 8468 & 9680 & 10214 & 9715 & 9935 & 9870 & 9410 \\
\hline Age not known & KEWL & 308 & 261 & 243 & 71 & 4 & 2 & - & - & 2 & - & - \\
\hline Males and Females & KEWM & 132298 & 122858 & 108279 & 116768 & 122484 & 128068 & 129253 & 127848 & 134077 & 133342 & 125419 \\
\hline Under 16 & KEWN & 114 & 80 & 66 & 84 & 134 & 113 & 125 & 141 & 130 & 159 & 124 \\
\hline 16 to 20 & KEWO & 30652 & 27761 & 24746 & 26444 & 26790 & 26381 & 25989 & 26981 & 28768 & 27668 & 23151 \\
\hline 21 to 30 & KEWP & 50473 & 46272 & 41094 & 44652 & 46252 & 47556 & 46993 & 45468 & 47788 & 48606 & 46045 \\
\hline Over 30 & KEWQ & 49829 & 47665 & 41437 & 45226 & 49285 & 54011 & 56140 & 55255 & 57387 & 56909 & 56099 \\
\hline Age not known & KEWR & 1230 & 1080 & 936 & 363 & 23 & 7 & 6 & 3 & 4 & - & - \\
\hline Companies & KEWS & 659 & 562 & 316 & 320 & 343 & 451 & 480 & 353 & 294 & 241 & 11 \\
\hline Total persons with charge proved ${ }^{2}$ & KEWT & 132957 & 123420 & 108595 & 117089 & 122827 & 128519 & 129733 & 128201 & 134363 & 133076 & 125430 \\
\hline
\end{tabular}

1 See chapter text.

2 Includes sex unknown. 


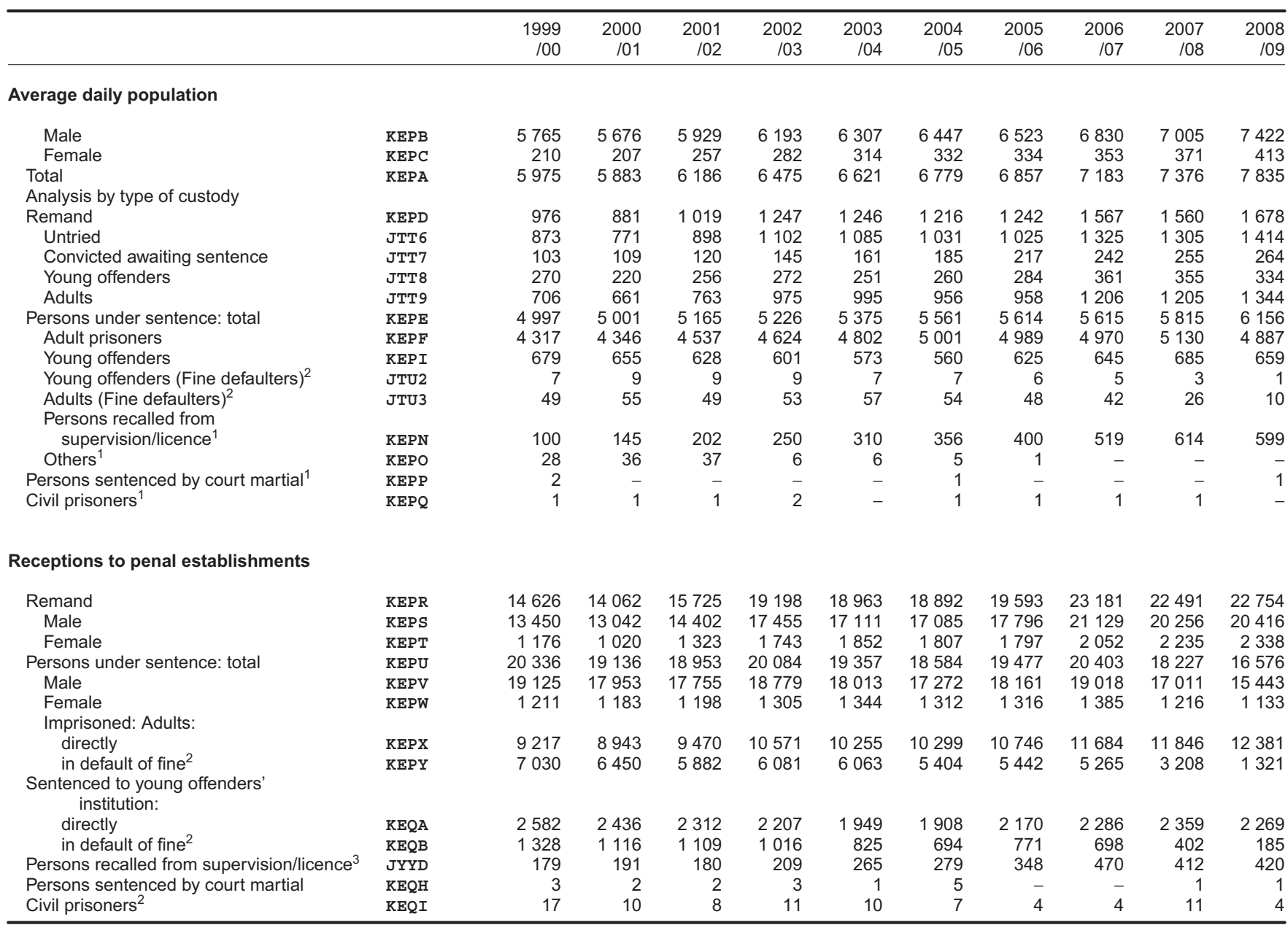

1 Persons recalled from supervision/licence and others are included in per- 2 Includes in default of compensation orders.

sons under sentence. Persons sentenced by court martial and civil prison- 3 Now covers all recalls from supervised release orders

ers are not included in persons under sentence.

\section{Expenditure on penal establishments ${ }^{1}$ \\ Scotland}

Years ended 31 March

$£$ thousand

\begin{tabular}{|c|c|c|c|c|c|c|c|c|c|c|c|c|}
\hline & & $\begin{array}{r}1998 \\
/ 99\end{array}$ & $\begin{array}{r}1999 \\
/ 00\end{array}$ & $\begin{array}{r}2000 \\
/ 01\end{array}$ & $\begin{array}{r}2001 \\
/ 02\end{array}$ & $\begin{array}{r}2002 \\
103\end{array}$ & $\begin{array}{r}2003 \\
104\end{array}$ & $\begin{array}{r}2004 \\
105\end{array}$ & $\begin{array}{r}2005 \\
106\end{array}$ & $\begin{array}{r}2006 \\
107\end{array}$ & $\begin{array}{r}2007 \\
108\end{array}$ & $\begin{array}{r}2008 \\
109\end{array}$ \\
\hline \multicolumn{13}{|l|}{ Departmental Expenditure } \\
\hline Manpower and Associated Services & KPHC & 144660 & 170347 & 160242 & 172490 & 168593 & 169784 & 181931 & 200742 & 199854 & 206298 & 214346 \\
\hline Prisoner and Associated Costs & KPHD & 18891 & 22930 & 23501 & 24652 & 23363 & 51070 & 42767 & 28582 & 41821 & 29408 & 29793 \\
\hline Capital Expenditure & KPHE & 23697 & 28918 & 24283 & 24955 & 36519 & 34617 & 72812 & 70406 & 81818 & 53564 & 100231 \\
\hline Gross Expenditure & KPHF & 187248 & 222195 & 208026 & 222097 & 228475 & 255471 & 297510 & 299730 & 323493 & 289270 & 344370 \\
\hline Less Receipts & KPHG & 8160 & 6668 & 8380 & 8194 & 3485 & 3298 & 3312 & 2872 & 2178 & 2034 & 2579 \\
\hline Net Departmental Expenditure & $\mathrm{KPHH}$ & 179088 & 215527 & 199646 & 213903 & 224990 & 252173 & 294198 & 296858 & 321315 & 287236 & 341791 \\
\hline \multicolumn{13}{|l|}{ Plus Annually Managed Expenditure } \\
\hline Capital Charges & DSJI & .. & .. & .. & 31341 & 40432 & 41728 & 48497 & 52840 & 41816 & 59498 & 89656 \\
\hline Total Net Expenditure & DSNX & 179088 & 215527 & 199646 & 245244 & 265422 & 293901 & 342695 & 349698 & 363131 & 346734 & 431447 \\
\hline
\end{tabular}


Thousands

\begin{tabular}{|c|c|c|c|c|c|c|c|c|c|c|c|c|c|c|}
\hline & & \multicolumn{2}{|c|}{ Old counting rules } & & \multicolumn{10}{|c|}{ New counting rules } \\
\hline & & 1996 & 1997 & & $\begin{array}{r}1999 \\
100\end{array}$ & $\begin{array}{r}2000 \\
101\end{array}$ & $\begin{array}{r}2001 \\
102\end{array}$ & $\begin{array}{r}2002 \\
103\end{array}$ & $\begin{array}{r}2003 \\
104\end{array}$ & $\begin{array}{r}2004 \\
105\end{array}$ & $\begin{array}{r}2005 \\
106\end{array}$ & $\begin{array}{r}2006 \\
107\end{array}$ & $\begin{array}{r}2007 \\
108\end{array}$ & $\begin{array}{r}2008 \\
108\end{array}$ \\
\hline Violence against the person & RVCP & 5.6 & 5.2 & RVCQ & 21.4 & 21.4 & 26.1 & 28.5 & 29.0 & 29.3 & 31.0 & 31.8 & 29.6 & 29.5 \\
\hline Sexual offences & RVCR & 1.7 & 1.4 & RVCS & 1.3 & 1.2 & 1.4 & 1.5 & 1.8 & 1.7 & 1.7 & 1.8 & 1.8 & 1.9 \\
\hline Burglary & RVCT & 16.1 & 14.3 & RVCU & 16.1 & 15.8 & 17.1 & 18.7 & 16.4 & 13.4 & 12.8 & 11.6 & 11.7 & 12.5 \\
\hline Robbery & RVCV & 1.7 & 1.7 & RVCW & 1.4 & 1.8 & 2.2 & 2.5 & 2.0 & 1.5 & 1.7 & 1.6 & 1.1 & 1.3 \\
\hline Theft & RVCX & 32.8 & 29.5 & RVCY & 37.0 & 36.9 & 41.7 & 41.9 & 35.7 & 31.1 & 29.5 & 27.8 & 24.7 & 26.2 \\
\hline Fraud and forgery & RVCZ & 4.1 & 3.8 & RVDA & 7.9 & 8.0 & 8.6 & 8.8 & 6.3 & 5.2 & 5.1 & 4.5 & 2.8 & 3.6 \\
\hline Criminal damage & RVDB & 4.8 & 4.7 & RVDC & 31.2 & 32.3 & 40.0 & 36.6 & 32.4 & 31.4 & 34.8 & 36.3 & 30.9 & 28.4 \\
\hline Offences against the state & RVDD & 0.4 & 0.5 & RVDE & 0.7 & 0.8 & 1.2 & 1.8 & 1.3 & 1.2 & 1.3 & 1.3 & 1.1 & 1.4 \\
\hline Other notifiable offences & RVDF & 1.2 & 1.1 & RVDG & 2.1 & 1.7 & 1.4 & 2.4 & 3.2 & 3.3 & 5.3 & 4.5 & 4.7 & 5.3 \\
\hline of which drug offences & RVDH & 1.1 & 1.0 & RVDI & 1.7 & 1.5 & 1.1 & 1.9 & 2.6 & 2.6 & 2.9 & 2.4 & 2.7 & 3.0 \\
\hline Total & RVDR & 68.5 & 62.2 & RVDS & 119.1 & 119.9 & 139.8 & 142.5 & 128.0 & 118.1 & 123.2 & 121.1 & 108.5 & 110.1 \\
\hline
\end{tabular}

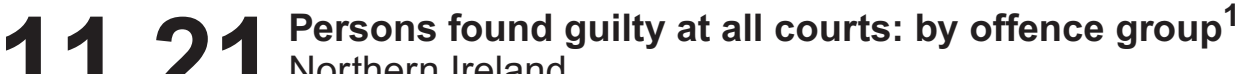 \\ Northern Ireland}

\begin{tabular}{|c|c|c|c|c|c|c|c|c|c|c|c|c|}
\hline & & 1996 & 1997 & 1998 & 1999 & 2000 & 2001 & 2002 & 2003 & 2004 & 2005 & 2006 \\
\hline Violence against the person & КYCT & 1597 & 1594 & 1596 & 1699 & 1858 & 1621 & 1790 & 1965 & 2012 & 2009 & 229 \\
\hline Sexual offences & KEVG & 184 & 130 & 128 & 90 & 130 & 112 & 84 & 108 & 137 & 136 & 16 \\
\hline Burglary & KYBW & 801 & 715 & 647 & 703 & 703 & 496 & 595 & 602 & 620 & 557 & 53 \\
\hline Robbery & KYBX & 161 & 166 & 134 & 129 & 122 & 121 & 152 & 192 & 159 & 135 & 14 \\
\hline Theft & KYBY & 2765 & 2596 & 2342 & 1995 & 2111 & 1831 & 1695 & 1803 & 1819 & 1819 & 172 \\
\hline Fraud and forgery & KYBZ & 467 & 491 & 426 & 476 & 403 & 398 & 362 & 314 & 359 & 330 & 33 \\
\hline Criminal damage & KYCA & 1076 & 1163 & 1043 & 931 & 1060 & 917 & 957 & 1034 & 1094 & 1168 & $12 \mathrm{~s}$ \\
\hline Offences against the state & KYCB & 147 & 165 & 198 & 178 & 174 & 158 & 215 & 274 & 252 & 270 & \\
\hline Other indictable ${ }^{2}$ & $\mathrm{KYCC}$ & 899 & 739 & 936 & 943 & 700 & 495 & 453 & 527 & 636 & 722 & \\
\hline Total indictable ${ }^{3}$ & KYCD & 8097 & 7759 & 7450 & 7144 & 7261 & 6149 & 6303 & 6819 & 7088 & 7146 & 7635 \\
\hline Summary ${ }^{4}$ & KYCE & 4402 & 4435 & 4062 & 3598 & 3967 & 3735 & 3453 & 3514 & 3622 & 3575 & 3645 \\
\hline Motoring $^{5}$ & KYCF & 18177 & 18770 & 15369 & 15782 & 15390 & 14466 & 14344 & 16342 & 17215 & 15534 & 15083 \\
\hline All offences & KYCG & 30676 & 30964 & 26881 & 26524 & 26618 & 24350 & 24100 & 26675 & 27925 & 26255 & 263 \\
\hline
\end{tabular}

1 See chapter text

21998 and 1999 figures include 'dangerous driving' (a triable-either-way offence).

3 From 2000, includes 'indictable-only' motoring offences.
4 Excludes motoring offences.

5 Prior to 2000, includes all motoring offences (except for note 2 above). From 2000 , includes summary and triable-either-way motoring offences.

\section{$1422 \begin{aligned} & \text { Juveniles found guilty at all courts: }{ }^{1} \text { by offence group } \\ & \text { Northern Ireland }\end{aligned}$}

\begin{tabular}{|c|c|c|c|c|c|c|c|c|c|c|c|c|}
\hline & & 1996 & 1997 & 1998 & 1999 & 2000 & 2001 & 2002 & 2003 & 2004 & 2005 & 2006 \\
\hline Violence against the person & $\mathrm{KYCH}$ & 75 & 49 & 97 & 73 & 77 & 66 & 82 & 75 & 78 & 146 & 152 \\
\hline Burglary & KYCI & 137 & 124 & 108 & 117 & 125 & 73 & 77 & 89 & 66 & 113 & 81 \\
\hline Robbery & KYCJ & 13 & 18 & 4 & 7 & 15 & 8 & 14 & 10 & 6 & 8 & 11 \\
\hline Theft & KYCK & 338 & 334 & 304 & 227 & 254 & 244 & 212 & 173 & 183 & 291 & 202 \\
\hline Fraud and forgery & KYCL & 14 & 11 & 4 & 10 & 2 & 9 & 3 & 7 & 2 & 6 & 7 \\
\hline Criminal damage & KYCM & 121 & 136 & 139 & 102 & 143 & 152 & 132 & 162 & 129 & 241 & 240 \\
\hline Offences against the state & KYCN & 6 & 10 & 11 & 12 & 8 & 10 & 20 & 26 & 18 & 19 & 40 \\
\hline Other indictable ${ }^{2}$ & KYCO & 24 & 10 & 20 & 17 & 10 & 12 & 7 & 19 & 22 & 46 & 44 \\
\hline Total indictable ${ }^{3}$ & KYCP & 732 & 700 & 699 & 577 & 638 & 575 & 553 & 566 & 511 & 879 & 785 \\
\hline Summary ${ }^{4}$ & $\mathrm{KYCQ}$ & 182 & 198 & 187 & 163 & 180 & 203 & 194 & 174 & 135 & 296 & 258 \\
\hline Motoring $^{5}$ & KYCR & 58 & 57 & 98 & 97 & 82 & 102 & 89 & 94 & 76 & 280 & 230 \\
\hline All offences & KYCS & 972 & 955 & 984 & 837 & 900 & 880 & 836 & 834 & 722 & 1455 & 1273 \\
\hline
\end{tabular}

\footnotetext{
1 See chapter text. For the purpose of criminal proceedings, prior to 30 August 2005, a juvenile refers to a person aged 10 years or more but under 17. From 30 August 2005, the youth justice system was extended to include those under the age of 18 . The number of juveniles convicted in
} 2005 and 2006 refers to those aged 10 years or more but under 18 .
21998 and 1999 figures include 'dangerous driving'.

3 From 2000, includes 'indictable-only' motoring offences.

4 Excludes motoring offences.

5 Prior to 2000 includes all motoring offences (except for note 2 above). From 2000, includes summary and triable-either-way motoring offences. 


\begin{tabular}{|c|c|c|c|c|c|c|c|c|c|c|c|c|}
\hline & & 1996 & 1997 & 1998 & 1999 & 2000 & 2001 & 2002 & 2003 & 2004 & 2005 & 2006 \\
\hline \multicolumn{13}{|l|}{ Magistrates court - all offences } \\
\hline Prison $^{1}$ & KYAO & 1003 & 989 & 996 & 1278 & 1356 & 1048 & 1107 & 1133 & 1101 & 977 & 1018 \\
\hline Custody Probation Order ${ }^{1}$ & EOG9 & & & & & & & & 7 & 7 & 9 & 12 \\
\hline Young offenders centre & KYAP & 443 & 430 & 326 & 243 & 191 & 209 & 288 & 395 & 456 & 416 & 366 \\
\hline Training school $^{2}$ & KYAQ & 147 & 148 & 136 & 13 & & & & & & & \\
\hline Juvenile Justice Centre order ${ }^{2}$ & OEUX & .. & .. & .. & 22 & 78 & 72 & 58 & 48 & 50 & 50 & 35 \\
\hline Total immediate custody & KYAR & 1593 & 1567 & 1458 & 1556 & 1625 & 1329 & 1453 & 1583 & 1614 & 1452 & 1431 \\
\hline Prison suspended & KYAS & 1722 & 1506 & 1025 & 1080 & 1247 & 1215 & 1278 & 1407 & 1469 & 1584 & 1692 \\
\hline YOC suspended & KYAT & 444 & 461 & 139 & 104 & 93 & 77 & 100 & 201 & 372 & 375 & 335 \\
\hline Attendance centre & KYAU & 91 & 66 & 55 & 14 & 20 & 37 & 84 & 91 & 108 & 127 & 132 \\
\hline Probation/supervision $^{3}$ & KYAV & 1134 & 1155 & 1473 & 1246 & 1096 & 1070 & 1005 & 974 & 991 & 977 & 1045 \\
\hline Community service order & KYAW & 591 & 561 & 622 & 678 & 726 & 587 & 643 & 623 & 647 & 628 & 597 \\
\hline Combination order & OEUZ & .. & .. & 38 & 7 & 48 & 24 & 36 & 96 & 78 & 106 & 133 \\
\hline Fine $^{4}$ & KYAX & 20614 & 21313 & 17956 & 18076 & 17716 & 16439 & 15968 & 17546 & 18520 & 17231 & 17311 \\
\hline Conditional discharge & KYAZ & 1679 & 1597 & 1538 & 1439 & 1286 & 1559 & 1497 & 1526 & 1524 & 1326 & 1093 \\
\hline Absolute discharge & KYBA & 509 & 424 & 303 & 223 & 242 & 209 & 163 & 201 & 183 & 148 & 129 \\
\hline Youth conference order ${ }^{5}$ & GGL 8 & .. & .. & .. & .. & .. & .. & .. & .. & 21 & 74 & 304 \\
\hline Community responsibility order & GGL9 & .. & .. & .. & .. & .. & .. & .. & .. & 1 & 32 & 71 \\
\hline Reparation order & J8FR & & .. & & & & & & & & & 1 \\
\hline Other & КYBC & 15 & 8 & 123 & 221 & 57 & 61 & 104 & 215 & 190 & 122 & 61 \\
\hline Total & KYBD & 29595 & 29925 & 25864 & 25733 & 25513 & 23417 & 23243 & 25554 & 26631 & 25035 & 25028 \\
\hline \multicolumn{13}{|l|}{ Crown court - all offences } \\
\hline Prison $^{1}$ & KYBE & 469 & 475 & 520 & 386 & 521 & 407 & 410 & 238 & 259 & 248 & 318 \\
\hline Custody Probation Order ${ }^{1}$ & $\mathrm{EOH} 2$ & & .. & & .. & .. & .. & & 331 & 332 & 370 & 416 \\
\hline Young offenders centre & KYBF & 106 & 111 & 63 & 67 & 32 & 42 & 23 & 51 & 47 & 41 & 38 \\
\hline Training school $^{2}$ & KYBG & - & 4 & 2 & - & .. & .. & .. & .. & .. & .. & .. \\
\hline Juvenile Justice Centre order ${ }^{2}$ & VQEV &.. & .. & - & - & - & - & 2 & - & - & - & 1 \\
\hline Total immediate custody & КYBH & 575 & 590 & 585 & 453 & 553 & 449 & 435 & 620 & 638 & 659 & 773 \\
\hline YOC suspended & KYBJ & 71 & 60 & 49 & 41 & 48 & 37 & 35 & 50 & 72 & 45 & 42 \\
\hline Attendance centre & KYBK & - & - & - & - & - & - & 1 & - & - & - & 1 \\
\hline Probation/supervision ${ }^{3}$ & KYBL & 49 & 47 & 70 & 43 & 68 & 48 & 49 & 63 & 93 & 79 & 91 \\
\hline Community service order & KYBM & 54 & 37 & 33 & 24 & 29 & 45 & 25 & 27 & 33 & 31 & 32 \\
\hline Combination order & ZAEP & .. & .. & 13 & 6 & 7 & 5 & 18 & 34 & 33 & 40 & 22 \\
\hline Fine $^{4}$ & KYBN & 39 & 40 & 25 & 20 & 40 & 38 & 32 & 49 & 108 & 57 & 51 \\
\hline Recognizance & Күво & 7 & 10 & 7 & - & 4 & 11 & 12 & 8 & 6 & 9 & 8 \\
\hline Conditional discharge & KYBR & 30 & 31 & 23 & 17 & 38 & 36 & 20 & 24 & 45 & 28 & 33 \\
\hline Absolute discharge & KYBS & - & 1 & 6 & - & 3 & - & 6 & 1 & 1 & 6 & 4 \\
\hline Youth conference order ${ }^{5}$ & GGM2 &.. & .. & .. & .. & .. & .. & .. & .. & - & - & 5 \\
\hline Community responsibility order & GGM3 &.. & .. & .. & .. & .. & .. & .. & .. & - & - & - \\
\hline Reparation order & J8FS & .. & .. & .. & .. & .. & .. & .. & .. & .. & .. & - \\
\hline Other & KYBU & 3 & 3 & 7 & 2 & 2 & 2 & 4 & 5 & 3 & 6 & 6 \\
\hline Total & KYBV & 1081 & 1039 & 1017 & 791 & 1105 & 933 & 857 & 1121 & 1294 & 1220 & 1335 \\
\hline
\end{tabular}

1 Custody Probation Orders cannot be separately identified from 'prison' sentences from 1998 to 2002 . Thus during this timeframe, figures for prison include custody probation orders.

2 The Juvenile Justice Centre order replaced the training school order from 31st January 1999.
3 Supervision orders were abolished with the introduction of the Criminal Justice (Children) Northern Ireland Order 1998.

4 From 2000, fine incorporates 'fine plus disqualification' and 'fine plus penalty points'

5 Refers to the number of youth conference orders completed.

Source: Northern Ireland Office: 02890527157 
Crime and justice

Prisons and Young Offenders Centres

Northern Ireland

Receptions and average population

Numbers

\begin{tabular}{|c|c|c|c|c|c|c|c|c|c|c|c|c|}
\hline & & 1997 & 1998 & 1999 & 2000 & 2001 & 2002 & 2003 & 2004 & 2005 & 2006 & 2007 \\
\hline \multicolumn{13}{|l|}{ Receptions: } \\
\hline \multicolumn{13}{|l|}{ Reception of untried } \\
\hline prisoners & KEOA & 2188 & 2284 & 2497 & 2197 & 1922 & 2337 & 2439 & 2440 & 2776 & 3193 & 2929 \\
\hline \multirow{2}{*}{\multicolumn{13}{|c|}{$\begin{array}{l}\text { Reception of sentenced prisoners: } \\
\text { Imprisonment under }\end{array}$}} \\
\hline & & & & & & & & & & & & 1123 \\
\hline Imprisonment in default & КЕОВ & 1002 & 949 & 963 & 1001 & 191 & 910 & 1032 & 915 & 960 & 1075 & 1123 \\
\hline of payment of a fine & KEOC & 1513 & 1530 & 1423 & 1261 & 1090 & 990 & 1143 & 1296 & 1437 & 1569 & 1425 \\
\hline \multicolumn{13}{|l|}{$\begin{array}{l}\text { Reception into Young } \\
\text { Offender Centres: }\end{array}$} \\
\hline \multicolumn{13}{|l|}{ Detention under sentence } \\
\hline of immediate custody & $\mathrm{KEOE}$ & 331 & 347 & 346 & 282 & 252 & 315 & 268 & 287 & 222 & 229 & 247 \\
\hline \multicolumn{13}{|l|}{ Detention in default of } \\
\hline payment of a fine & KEOF & 366 & 385 & 417 & 389 & 303 & 250 & 310 & 351 & 377 & 382 & 299 \\
\hline Total & KEOG & 697 & 732 & 763 & 671 & 555 & 565 & 578 & 638 & 599 & 611 & 546 \\
\hline \multicolumn{13}{|l|}{ Daily average population: } \\
\hline Unconvicted $^{3}$ & KEON & 376 & 383 & 377 & 317 & 272 & 347 & 393 & 456 & 450 & 531 & 531 \\
\hline Convicted $^{4}$ & KEOP & 1256 & 1124 & 867 & 751 & 638 & 679 & 767 & 818 & 851 & 902 & 935 \\
\hline Total & KEOM & 1632 & 1507 & 1244 & 1068 & 910 & 1026 & 1160 & 1274 & 1301 & 1433 & 1466 \\
\hline
\end{tabular}

1 Includes those detained under Section 73 of the Children and Young Per- 3 Prisoners on remand or awaiting trial and prisoners committed by civil process sons (NI) Act 1968

4 Includes those sentenced to immediate custody and fine defaulters.

2 Non-criminal prisoners including those imprisoned for non-payment of

Sources: The Northern Ireland Prison Population in 2007;

maintenance, non-payment of debt, contempt of court or are being held un-

Northern Ireland Office: 02890527534

der the terms of an Immigration Act. 